\title{
Identification of Low-engaged Learners in Robot-led Second Language Conversations with Adults
}

\author{
OLOV ENGWALL and RONALD CUMBAL, KTH Royal Institute of Technology \\ JOSÉ LOPES, Heriot-Watt University \\ MIKAEL LJUNG and LINNEA MÅNSSON, KTH Royal Institute of Technology
}

\begin{abstract}
The main aim of this study is to investigate if verbal, vocal, and facial information can be used to identify low-engaged second language learners in robot-led conversation practice. The experiments were performed on voice recordings and video data from 50 conversations, in which a robotic head talks with pairs of adult language learners using four different interaction strategies with varying robot-learner focus and initiative. It was found that these robot interaction strategies influenced learner activity and engagement. The verbal analysis indicated that learners with low activity rated the robot significantly lower on two out of four scales related to social competence. The acoustic vocal and video-based facial analysis, based on manual annotations or machine learning classification, both showed that learners with low engagement rated the robot's social competencies consistently, and in several cases significantly, lower, and in addition rated the learning effectiveness lower. The agreement between manual and automatic identification of low-engaged learners based on voice recordings or face videos was further found to be adequate for future use. These experiments constitute a first step towards enabling adaption to learners' activity and engagement through within- and between-strategy changes of the robot's interaction with learners.
\end{abstract}

\section{CCS Concepts: • Human-centered computing $\rightarrow$ Empirical studies in HCI;}

Additional Key Words and Phrases: Robot-assisted language learning, user engagement, speech emotion recognition, facial emotion expressions

\section{ACM Reference format:}

Olov Engwall, Ronald Cumbal, José Lopes, Mikael Ljung, and Linnea Månsson. 2022. Identification of Lowengaged Learners in Robot-led Second Language Conversations with Adults. ACM Trans. Hum.-Robot Interact. 11, 2, Article 18 (February 2022), 33 pages.

https://doi.org/10.1145/3503799

\footnotetext{
This work was supported by the Swedish Research Council under Grant 2016-03698 and Marcus and Amalia Wallenberg foundation under grant MAW 2020.0052.

Authors' addresses: O. Engwall and R. Cumbal, KTH Royal Institute of Technology, Department of Speech, Music and Hearing, Lindstedtsv. 24, Stockholm, Sweden, SE-10044; emails: \{engwall, ronaldgc\}@kth.se; J. Lopes, Heriot-Watt University, Edinburgh, United Kingdom; email: JD.Lopes@hw.ac.uk; M. Ljung and L. Månsson, KTH Royal Institute of Technology, Stockholm, Sweden; emails: \{milju, lman\}@kth.se.
}

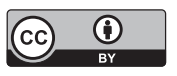

This work is licensed under a Creative Commons Attribution International 4.0 License.

(C) 2022 Copyright held by the owner/author(s).

2573-9522/2022/02-ART18

https://doi.org/10.1145/3503799 


\section{INTRODUCTION}

Adaptation is a key to successful human-human interaction and humans learn to adjust their interaction to the context, the interlocutor, and the information that is to be transferred. This is, in particular, true for tutor-learner interactions: Teachers adopt pedagogically adequate presentation styles to the level and engagement of their students, [17] and native speakers may modify (e.g., speaking rate, intonation) and simplify (e.g., regarding vocabulary) their utterances to facilitate understanding for less proficient second language (L2) speakers [14]. Such adaptation is a challenging task for educational robots, since it requires that they become aware that a change is required, then determine what type of change is needed and how to achieve this change. The effort is nevertheless worthwhile, as it has been shown that adaptation to users' engagement is essential for their long-term motivation to interact with a system [8] and further that adaptive social robots can increase language learners' emotional rapport to the robot [28] and even re-motivate language learners who have become disengaged [53].

We here concentrate on learner signals that indicate that an adaptation is necessary in L2 conversation practice of Swedish with pairs of adult learners, led by the anthropomorphic robotic head Furhat. The primary interest of the study is to investigate, with experiments using previously collected data [24], if learners that are identified through verbal, vocal, or facial information as being low-engaged during the conversation-i.e., are less active or display low emotional engagement (see further the definition of social-task engagement below)-also rate the robot and the practice lower post-session. If so, observations of low engagement are connected to the learners' own perception of their engagement. A second aim is to analyse if low learner activity and emotional engagement are more frequent with some of the robot's four interaction strategies, as these are more or less inclusive and inviting towards the learners. The strategies respectively aim for three-party interaction, sequential interactions with one learner at the time, robot-encouraged learner-learner interaction, and robot semi-monologues.

The three-party setting with two learners and a robot was inspired by spoken practice in the so-called language cafés, and surveys and interviews with language learners and teachers [24, 25], which concluded that a practice set-up in which learners interact with each other is beneficial both pedagogically (as they can support each other linguistically) and technologically (as the learners may get adequate practice even in cases of communication problems with the robot). The multiparty setting, however, introduces additional requirements of monitoring and adapting also to the learner that the robot is currently not focusing its attention on. As a special case, we therefore investigate if engagement levels can be automatically classified not only for the active participant (which is the standard procedure), but also for the currently non-active participant.

Engagement may be defined in different ways, in particular, both as the act of being involved in an activity and as being emotionally committed to this activity. In addition, one may distinguish between task engagement, social engagement, and social-task engagement [13]. Task engagement is created through a balance between the challenge of the [explicit] task and the learner's skill, and it is manifested through attention and concentration with respect to the learning task [22]. Social engagement is created by a sociable and friendly interaction between the participants, and it is manifested through the participants' willingness to establish and maintain the connection with the other participants $[49,54]$. Social-task engagement refers to the combination of the two above, i.e., active involvement and emotional engagement in the collaborative interaction to solve a task [13]. With an explicit task, it may be possible to consider task and social engagement separately [13], but we here need to consider joint social-task engagement, since the task is to maintain a spoken social interaction, and engagement hence covers both the interest in practicing the linguistic task and in interacting with the robot. 
We consider three observable measures of engagement during the conversations that all can indicate an interest in both the task and the interaction with the robot-the learners' verbal activity, their emotional vocal signals and their emotional facial expressions-and two relative measures of the learners' subjective opinions post-session: Their ratings of the practice from a learning perspective and the robot from a social perspective (regarding how personal, friendly, conversationally competent, and human-like the robot is). We want to investigate if the observed indicators of engagement during the conversation may be related to the learners' ratings of learning (linked to task engagement) or the robot's social competencies (linked to social engagement).

For the multi-party setting, with one robot and two learners, it is further important to note that whereas some human-robot interaction (HRI) multi-party studies [41,61] use the term "engaged" to refer to the active or addressed user, our definition refers to how interested the learner is in taking part in the interaction or being an active listener. Thus, an active speaker can have a low engagement, in terms of little verbal activity and/or low emotional interest for the interaction, and a non-active speaker (the listener) can be emotionally and task engaged. This further signifies that the activity and emotional engagement of the individual participants, rather than the engagement of the group (e.g., [46]) need to be measured, since the aim is for the robot to be able to react to differences between learners in the same conversation and adjust the verbal (e.g., speaking rate, interaction style, and utterance complexity) or non-verbal (e.g., gaze and head turns, visual feedback signals, and turn distribution) output towards each of the learners.

The main research question $(\mathrm{R})$ Can learners with low social-task engagement in robot-led second language conversation practice be identified through their verbal activity, their voice features, and their facial expressions? is investigated based on an offline analysis of the relationship between the learners' interaction data (verbal transcriptions and audio and video recordings) and their postsession ratings of the robot.

The aim is hence to find objective indicators in the conversations allowing to identify learners who have a more negative subjective opinion of the practice and/or the robot post-session, in order to use such indicators in the future to be able to adapt the robot's interaction strategy during the conversation to better engage these learners. To investigate the main research question, five sub-questions are addressed. The first three are related to how the robot's interaction strategy influences the observable measures of engagement during the conversation, i.e., the learners' verbal activity (R1, Section 5), emotional vocal expressions (R2, Section 6), and facial expressions (R3, Section 7). In addition, we investigate if facial expressions may be used to determine how emotionally engaged the currently non-active learner is (R4, Section 7.2), and if standard methods for emotion detection agree with manual annotation of engagement (R5, Sections 6 and 7.2). A schematic overview of the experiments and their use of different data sources is provided in Figure 1.

The hypotheses are that learners with low verbal activity or low emotional engagement expressed in voice features or facial expressions rate the robot's social capabilities and/or the practice effectiveness lower $(\mathrm{H})$; that the different robot interaction strategies give rise to different levels of the observable measures of engagement: learner verbal activity (H1), emotional vocal expressions $(\mathrm{H} 2)$, and emotional facial expressions (H3); that non-active learners display facial expressions that signal their emotional engagement (H4) and that existing tools for automatic emotional engagement detection have a reasonable agreement with manual annotation (H5).

To set the basis for these experiments, related work on engagement in different types of human-robot interaction (Section 2) is first described, then general methods for engagement analysis (Section 3), before the previously conducted user experiment on robot-led conversation practice in which the data were recorded is presented (Section 4), the three different analyses are reported (Sections 5-7), and the results are discussed (Section 8). 


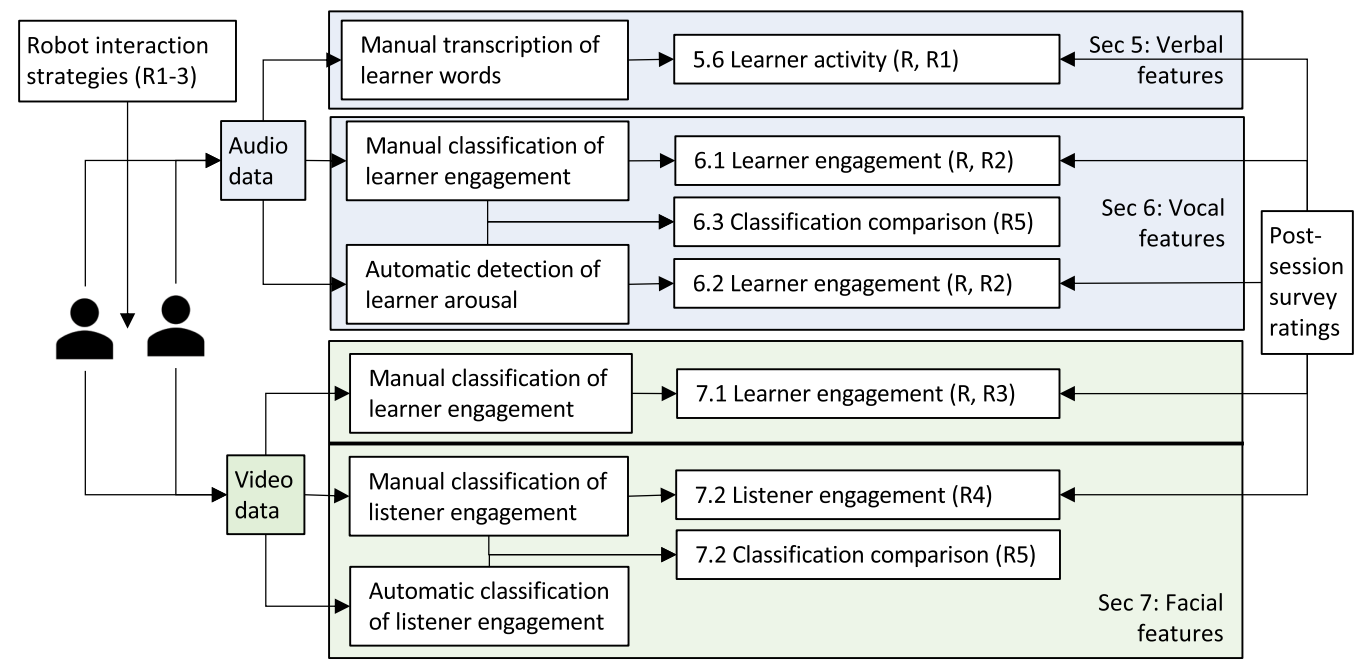

Fig. 1. Overview of the different sections of the article, the research questions and the use of audiovisual data to explore the relationship between verbal, vocal or facial features, and learners' post-session ratings.

\section{PREVIOUS WORK ON USER ENGAGEMENT IN HRI}

In previous work, activity, emotional engagement, and interest have been measured using interaction time [54], gaze patterns and mutual gaze [12, 41, 48, 50, 54], facial expressions [12, 41, 59], head-pose [48], hand-face actions [12], speech [55], backchannels [50], turn-taking [50], physiological data $[5,44]$, or self-rating $[5,54]$.

Gazepatterns have long been a primary source of information on engagement, and mutual gaze has been investigated between human users and, e.g., a penguin robot giving instructions [54] and virtual agents [48]. More and more, video-based analysis of facial expressions [12, 59], sometimes including additional information, such as hand gestures in the face region, has come to dominate both manual labeling and automatic classification. It is further common to combine several modalities, such as gaze, backchannels, and interaction data (e.g., [50]).

For the present study, engagement in multi-party settings and in educational interaction, especially in language learning, are of primary interest, as they add additional requirements on interaction with, respectively, several users and the learning material. With a few exceptions $[24,32,37]$, most previous work on robot-assisted language learning has been carried out with children, whereas the present study is with adult learners, who have different requirements and preferences regarding the robot's appearance, role, and interaction [23]. They may further both react differently to the robot's behaviour and display their reactions in a different manner than the child learners who have been studied in previous work on engagement in robot-assisted language learning $[1,2,16,28,53]$. The present study hence extends previous work by considering another learner group (adults), which is more heterogeneous (in the native language, cultural background, and age).

\subsection{User Engagement in Multi-party Human-robot Interaction}

In multi-party settings, it is important to be socially aware of all [potential] participants in the interaction, and it has been shown that robot awareness of the users' engagement has a positive effect on their experience [41], in particular, regarding user ratings of how human-like and 
intelligent the robot is and the smoothness of the interaction. Being socially aware in a multiparty setting requires that the robot can monitor the affective state of the non-active participants as well as the active and is able to model their levels of engagement separately. It has been shown that a different model may be needed for engagement classification in multi-party settings [40] and engagement estimation may need to be performed on different features than in interaction with one user. Acoustic cues, which are used in Section 6, have previously been used to determine participant affective state (positive, neutral, sadness, fear, and anger), valence and activation in interactions between pairs of children and a robot [55]. Audiovisual information has been used to determine if an on-looker is interested in joining the game between a peer and a robot [38], and a study on multi-party all-human group interviews [45] highlighted gaze changes as important cues to determine how engaged a non-speaking participant is. Such visual cues are further examined in the listener engagement analysis in Section 7.2.

Another study [4], in which children played a game together with either a peer or the robot Sammy J, resulted in two findings of interest for the present work: Firstly, there was no general correlation between the annotated engagement levels of the two children in the pair: Their relative engagement levels were similar over the interaction in some pairs, but in others they were quite different. Secondly, the children's engagement during the interaction with the robot varied substantially less over time than when they were interacting with a peer. This suggests that there may well be differences in engagement level between the two learners in the conversation practice described below, and that there may be differences between robot strategies aiming for predominantly robot-learner or learner-learner interaction.

\subsection{Learner Engagement in Robot-assisted Language Learning}

Users' engagement and affective state influence general human-robot interaction, but they are even more important in educational situations, as affective states have an effect on the learning outcome [20]. Since robot-assisted learning is a relatively new area of research, there are only a few studies on engagement in robot-led language practice [1, 2, 16, 28, 53].

One study [28] used a facial expression software to determine child learners' affective state and the robot then responded to this with different non-verbal behaviours. The study found that these non-verbal robot responses influenced the learners' emotions towards the practice (valence), thus illustrating both how affective state detection can be used in robot-assisted language learning and that learners respond to the robot's behaviour. Another study [53] specifically focused on if a robot tutor that reacts to child learners' low engagement levels (detected by a human Wizard-of-Oz) can re-engage the children in the practice and found that this was successful in a majority (76\%) of the interactions. The present study has a related theme of responding to the learners' affective state and adapting for learner re-engagement, but focuses on the earlier step of enabling autonomous detection of low engagement or negative affective state.

In a study [16], in which learner engagement was determined through manual coding, it was demonstrated that tutor feedback corresponding to human teachers' preferred responses regarding if an answer was correct or not resulted in significantly higher child learner engagement than if no or fixed binary feedback was given. Since there are no correct or incorrect learner answers in the social conversations in this study, the main transferable insight is that learner engagement may be influenced by if the robot responds to learner utterances in a pedagogically sound manner or not.

Another question concerns if a robot that is more socially competent or provides more positive feedback enhances learning. Results are mixed between studies that find no difference between different feedback strategies [16] and those that do [2]. The latter study compared positive 
(appraisal), neutral, and negative (critical) feedback and found not only that positive feedback resulted in higher learner engagement (when measured as a duration of gaze directed at the robot) but also significantly more words retained [2].

It should be noted that affective learner signals and states are often complex. For example, smiles-of different types-occur both when the learners are pleased with the interaction and when they are frustrated, and this difference may be detected automatically [31]. Moreover, a review of emotion recognition in computer-assisted learning [20] showed that affective states such as concentration, frustration, confusion, and boredom are much more frequent in this setting than the set of basic emotions [21]. Two early studies on detection of, respectively, learner frustration (in a laboratory setting) [36] and confidence, frustration, excitement, and interest in the classroom setting [5] used facial video images, interaction data, skin conductance sensor, a pressure-sensitive mouse and a posture-sensing chair, and achieved $70 \%-80 \%$ correct classification of learners' affective state; hence, demonstrating the possibility to achieve high levels of prediction, but also that this may require a complex set of input data. Another study [9] with students studying physics problems used only video images for classification of facial expressions into bored, confused, frustrated, engaged and delighted with a Bayes network, and achieved an accuracy of $54 \%$, which is substantially lower than the more complex input data in $[5,36]$.

Consequently, as covered in Section 3, it is important to determine what type of data to use to attempt detecting engagement and which categories of engagement are relevant for educational human-robot interaction.

\section{METHODS FOR ANALYSING USER ENGAGEMENT}

This section gives a short summary of general methods for detection of engagement or emotions based on what users are saying (verbal information), how they are saying it (prosodic and other non-linguistic vocal information), and the facial expressions when saying it (image-based information). A more complete introduction is available in, e.g., [62]. The section also discusses considerations and choices for engagement analysis in the present study.

\subsection{Data Recordings and Labeling}

The present study is based on recordings with real engagement in a real-life setting; thus, following the shift in emotion and engagement detection studies from acted emotions in laboratories $[31,36,54]$ to real emotions in the wild $[9,12,19,40,55,62]$. This shift is important, since acted emotions may not be representative and studies have shown, e.g., that smiles in real and acted frustration differ substantially [31]. Real-life recordings with portable equipment (e.g., head-mounted microphone, web-cam in rooms with background noise), may, however, result in lower classification results, since the recorded data may be noisier than in laboratory recordings (with soundproofed room, broad frequency spectrum stationary microphone, and high-resolution video). Another consequence is that the real-life data need annotations, by one or several annotators (e.g., two in [7, 40, 55]; three in [4, 12]; unspecified number in $[9,18,50])$. In this study, one annotator labelled all audio recordings in Section 5, three annotators labelled an equal number of video recordings in Section 7.1, after ensuring high inter-rater agreement by using a common reference protocol, and nine different annotators provided up to five labels per video sequence in Section 7.2.

\subsection{Feature Selection}

The basic principle for engagement or affective state classifications is that the verbal, acoustic and/or visual input from the user is described using a set of features that can be compared with measures of absolute or relative engagement (rule-based) or through correspondence with an annotated training set (data-driven classification). 
For verbal classification, the features are often words/lemmas, and their emotional charge is determined through linguistic or psychological studies, in which subjects report their emotional reactions to different words, along different dimensions, such as valence (happy to unhappy), arousal (calm to excited), and dominance (in control to dominated) [57]. The verbal analysis in Section 5 is more restricted, since its most important contribution in the specific context of spoken L2 practice is to identify learners with low verbal activity (few and/or short utterances) and link these with their post-session ratings.

For acoustic-based classification, features may be determined through knowledge of what is important for human perception of emotions: prosodic features (fundamental frequency, duration, energy), voice quality features, speech disfluencies, non-linguistic events (laughter, inspiration, and expiration) and other vocal sounds $[11,18,55]$, or they may be automatically extracted from the speech signal by the classifier [7], as in Section 6.

For video-based classification, different feature sets have been used (e.g., image pixel-based), but the currently dominating strategy is to parameterise facial expressions in the Facial Action Coding System, using Facial Action Units (FAU). The geometric configurations of facial expressions (such as eyebrow raising, lip corner pulling) described by FAU can be related to facial emotion expression labels or learner interest $[48,59]$. However, since it is inefficient to use the full set of FAU, a selection of FAUs is often made, either manually, based on the intuition of which facial features are important for human perception of emotions, or empirically (and often automatically in state-of-the-art machine learning software [43]) based on preliminary results on a small sub-set (as in Section 7.2).

\subsection{Classification of Engagement}

Verbal-based classification of emotions and engagement can be performed using, e.g., bag-of-words representations of utterances classified according to how frequently the contained words have been labelled as belonging to a certain emotion class, using Point-wise Mutual Information [52]. As one example, Devillers et al. [19] used a similarity measure between an utterance and the normalised log-likelihood ratio of emotion together with task-specific models to classify utterances according to four emotions, using both lexical and prosodic cues on emergency call center dialogues. They found that lexical cues outperformed prosodic cues ( $78 \%$ vs. $60 \%$ correct classification). The present work instead uses classifications based on intra-dataset statistical measures (identifying conversations with few or short learner utterances).

Acoustic and visual classification of engagement in previous work has used machine learning algorithms such as Support Vector Machines (SVM) [19, 31, 40, 55, 60], Decision Trees (such as Random Forests) [9, 50], Conditional Random Fields [26, 31], K-nearest neighbours [44], Hidden Markov Models [31, 59, 60], Gaussian Model [26, 36, 38], Bayesian network [9], or an Artificial Neural Network. The acoustic-based classification in Section 6 employs a bidirectional recurrent neural network for classification of emotional arousal (separating interactions with low learner emotional arousal from those with high), whereas the video-based classification in Section 7.2 utilises support vector machines to classify three engagement levels from FAU.

\section{ROBOT-LED CONVERSATION PRACTICE EXPERIMENT}

This work focuses on engagement in a three-party L2 conversation practice setting with a social robot. The background and the implementation is described in detail in [24], but in summary, we use the well-established language café concept of practising L2 spoken interaction through a social conversation with peer learners and one or several native speakers as moderators, but replace the human moderator by the Furhat robot. The benefits of this setup are firstly that the robot can guide learners' spoken practice by taking initiative in the interaction and provide examples of utterance 


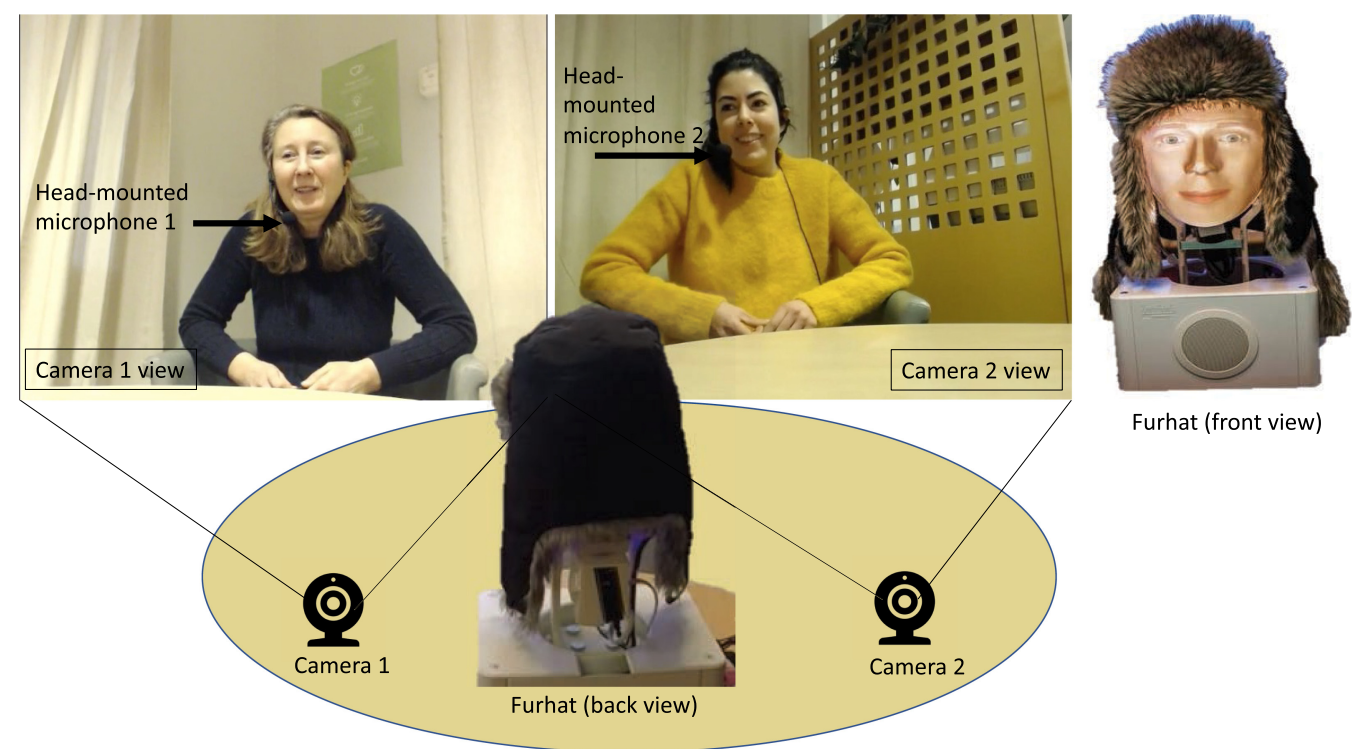

Fig. 2. Left: Set-up for the user experiment with Furhat and two web-cameras placed on a café table facing two learners, who each have a head-mounted microphone. Right: Front view of Furhat as seen by the learners.

construction at a higher proficiency level, and secondly that the peer learners may support each other with communication problems due either to their own proficiency level or weaknesses in the robot's verbal capabilities (e.g., understanding learners or rephrasing utterances).

The Furhat anthropomorphic robotic head [3], shown in Figure 2, has a computer-animation face back-projected on an interchangeable 3D-printed mask, and can hence display realistic facial signals (such as lip, eye and eyebrow movements, and emotional expression) that could be used to respond to learner engagement in the future (no responses to different learner engagement levels were implemented in the present study). The motor-servo neck allows the robot to distribute the turn between the learners in a natural way. Since the robot consists of head and neck only, it does, on the other hand, not display any other body gestures than facial expressions and head movement.

Our previous study focused firstly on prerequisites for robot-led conversation practice, based on a survey with language café moderators and observations of human-led sessions, secondly on the implementation of different distinct interaction strategies for the robot (see Section 4.1) and thirdly on collecting audiovisual data that could be used for further development and interaction studies. The present study is based on the material that was collected during this previous user study (see Section 4.2) and expands it through the specific focus on learner engagement.

\subsection{Robot Interaction Strategies}

We investigated the four different robot interaction strategies described briefly below, and in more detail in [24], which also provides interaction examples for the four different strategies. The strategies were maintained throughout one conversation and differed in terms of learner-robot initiative and learner-robot focus as

Narrator-Robot initiative and robot focus, with the robot being the most active in transferring information to the learners. The robot is narrating about himself or Sweden (learners listening); asking quiz questions about Sweden (learners answering and collaborating); and leading a robotcentered dialogue (learners responding). Narrator is of interest for the study on participant en- 
gagement since, on one hand, robots' background stories are important for user engagement [8], but on the other hand, the lack of participant initiative can lead to disengagement.

Interviewer-Robot initiative and learner focus, with the robot asking each learner individual questions about, e.g., background and interests, before switching to the other learner with similar questions (one learner active, one listening). Interviewer is interesting for this study, since there may be substantial differences in engagement between the learners depending on how the robot's attention (number and length of turns) is distributed.

Interlocutor-Balanced initiative and focus, aiming for a three-party interaction and frequent shifts of robot attention between the learners. Mix of questions to one learner or both, requests for peer comments, robot narrating and answering about his own views, and preferences (three-party interaction). Interlocutor hence has a higher amount of clear attention shifts between the learners than the other strategies, and thus allows to investigate how engaged the non-addressed learner is by the interaction between the robot and the peer.

Facilitator-Learner initiative and learner focus, with the robot encouraging learners to interact with each other by asking the learners to suggest topics, to elaborate their answers and to comment on each others' narratives. Since Facilitator transfers much of the interaction to that between the peers, the learners' engagement will depend on how interested they are in talking to each other, which may be compared to their interest in talking to the robot with the other strategies.

Each of the robot interaction strategies had its own predefined, but semi-flexible dialogue network, i.e., all robot utterances and the 10 alternative transitions from one utterance to the next were predefined, but as alternatives were available for each transition and since different topics were interconnected, the dialogue flow differed not only between different interaction strategies, but also between sessions with the same robot strategy. An example of the robot's verbal and visual interaction is available at https://www.kth.se/profile/engwall/page/robot-video (N.B. the video was recorded for reference purposes only and video and audio quality is therefore inferior to that of the recordings analysed in this study).

\subsection{User Study Data}

We carried out a user experiment with a Wizard-of-Oz setup, in which a total of 33 language learners (18 women and 15 men) in classes of Swedish for immigrants (at B1 to B2 level, according to the Common European Framework of Reference) interacted in pairs with the robot. Students in Swedish for Immigrant courses have a large variety in background, and in the current study, the age distribution was 20 - 54 years old (with mean age 32 years); the set of first languages among the subjects were Arabic (10, from Syria, Egypt, Iran, and Iraq), Spanish (3), Italian (2), Polish (2), Russian (2), Ukrainian (2), Chinese, Croatian, Dari, Filipino, French, Greek, Kurdish, Persian, Portuguese, Punjabi, Somali, and Tigrin; and the subjects' educational level ranged from basic education to university degree. Since the subjects are representative of the target group for the robot-led conversation practice, this heterogeneity is intentional: The engagement detection needs to work for a varied learner group.

The subjects were introduced to the study using an informed consent form describing that they would participate in four conversations with the robot and that the goal was firstly for them to practice Swedish and secondly to assist in the development of the robot, but they were not otherwise instructed how to interact with the robot or told that the robot would use different conversation strategies.

Study set-up: The intention was a within-subjects design, i.e., that all subjects should experience all four robot settings in four different conversations, to be able to perform intra-subject comparisons of interaction and user ratings of the robot personalities. However, since conversations three and four were scheduled for the second or third day of the user experiment and eight subjects did 
Table 1. Number of Subjects Experiencing Different Combinations of Robot Interaction Strategy and Conversation Order, Total Number of Conversations Per Strategy $(\Sigma)$, and the Overall Mean Ratings for Each Robot Strategy

\begin{tabular}{l|ccccc|ccccc}
\hline & \multicolumn{4}{|c}{ Conversation } & \multicolumn{5}{c}{ Rating (0-5) } \\
Strategy & 1st & 2nd & 3rd & 4th & $\Sigma$ & Learning & Friendly & Personal & Conversational & Human-like \\
\hline Narrator & 10 & 8 & 8 & 2 & 28 & 3.4 & 3.0 & 2.7 & 2.9 & 2.8 \\
Interviewer & 7 & 6 & 6 & 3 & 22 & 3.4 & 3.5 & 3.1 & 3.4 & 2.8 \\
Interlocutor & 4 & 10 & 2 & 8 & 24 & 3.1 & 3.3 & 2.6 & 3.0 & 3.0 \\
Facilitator & 9 & 8 & 4 & 5 & 24 & 3.3 & 3.3 & 2.6 & 3.0 & 2.7 \\
\hline
\end{tabular}

not come to class on these days, an additional five subjects were recruited to participate in two dialogues in order to allow for the returning subjects to complete their four sessions. Total 19 subjects hence participated in four dialogues, 1 in three and 11 in two, resulting in a total of 100 sets of recordings from 50 conversations.

The intention was further that the number of and order of conversations with each robot strategy should be balanced, i.e., the same number of conversations with each strategy as first, second, third, and fourth conversation and in total. Due to the subject drop-out, the distribution became as shown in Table 1. For each learner, the first two conversations were with one peer, and the last two were with another, in order to investigate how peer familiarity influenced the interaction and to avoid repetition of conversation topics with the same pair of learners.

Video recordings were made of each learner using a GoPro directed towards their face and upper body (see Figure 2). These video recordings are here used to analyse facial expressions of engagement level and to train and perform automatic detection of listener engagement.

Audio recordings were made with one head-mounted microphone for each learner. As the recording ran for the entire session, the recordings also include utterances by the robot and the peer, which need to be filtered out in post-processing. This was done using a sound level threshold combined with manual adjustments. The audio recordings are here used to determine learner engagement based on voice quality.

Transcriptions of the learners' utterances were generated through manual annotation at the word level, i.e., excluding hesitations and mispronunciations. These transcriptions allow to analyse the number of utterances and their lengths in the number of words.

Dialogue logs recorded the robot's actions and utterances and if the robot was looking at the right or left participant or at both. These logs, used for the experiment in Section 7.2, may thus be used to determine which learner is currently addressed by the robot and who is the listener.

Survey answers were collected after each conversation, using a web-based Likert-scale questionnaire, in which the learners rated the session from a learning perspective (Learning, with 0 being "Poor" and 5 "Excellent"), the robot's friendliness (Friendly, with 0 being "Unfriendly" and 5 "Very friendly"), level of personality (Personal, with 0 being "Keeping a distance" and 5 "Too personal"), quality of conversational behaviour (Conversational, with 0 being "Extremely poor" and 5 "Excellent"), and human-likeness as a conversational partner (Human-like, with 0 being "Extremely machine-like" and 5 "As a human"). The ratings are considered to indicate the learners' own view of their social-task engagement level, with Learning to some extent being more closely related to task engagement, while Friendly, Personal, Conversational, and Human-likeness are more related to social engagement. The detailed analysis of the survey answers is provided in [24], where they were used to compare how learners assessed different interaction strategies depending on factors such as language level, gender, age, cultural background, and the order in which the strategy was experienced. A general finding was that Interviewer was ranked the highest by the subjects who 
experienced all four strategies. However, ratings differed substantially depending on different factors, not the least gender and linguistic level and over the group of all subjects, the average ratings, shown in Table 1 were rather similar between interaction strategies.

In this study, the survey answers are instead linked to the analyses of interaction within the conversations, to investigate if the ratings are influenced by low verbal activity or emotional engagement. We identify measures, and their thresholds, that could indicate that a learner has a low social-task engagement. For each of the measures in Sections 5-7.2 thresholds were set based on two choices. Firstly, since there is no universal level of low activity or engagement, two approaches to set the thresholds were considered, either activity or engagement level relative the subject group (finding e.g., the $25 \%$ least active learners) or absolute levels (finding observed low levels of the measure). The latter approach was selected, since, from an application point of view, the primary goal is to detect learners with low verbal activity or engagement regardless of how active their peers are. Secondly, each threshold value for a given measure should be the same for all post-session survey categories, rather than setting different thresholds for different categories. This choice is again motivated by the application point of view, since a low engagement detector would need to function with one common threshold value, rather than ones specific for each survey category. Thresholds were then selected, using a search in the range of lower values for each measure, so that the separation of low-engaged learners from the rest of the group resulted in the most significant results, i.e., primarily the highest number of survey categories with significant differences or the lowest p-values when several thresholds would give the same number of significant differences (for the same or different categories). This signifies that the thresholds presented below are not the only ones resulting in significant differences, but the ones that were determined to be most salient.

For the data analysis we first performed Kolmogorov-Smirnov goodness-of-fit hypothesis tests and determined that the datasets were not normally distributed, and hence used non-parametric significance tests for the tests below: Kruskal-Wallis; hereafter KW, for the tests of differences between robot interaction strategies and Mann-Whitney U-test; hereafter, MW, with HolmBonferroni correction for six repeated comparisons, in the posthoc tests to identify pairwise significant differences between interaction strategies (hence adjusting the significance level to $\alpha_{1}=0.0083$, $\alpha_{2}=0.01, \alpha_{3}=0.0125, \alpha_{4}=0.0167, \alpha_{5}=0.025$, and $\alpha_{6}=0.05$ for the ranked $\mathrm{p}$-values, with the number of samples for each interaction strategy given in Table 1). MW was also used for the significance tests of differences in post-session ratings by low-engaged learners compared to others. This categorical test is the most appropriate, since we would like to determine if the category of low engaged learners differ in their ratings from the rest of the learner group, rather than investigating if there is a general relation between engagement level and ratings.

\section{VERBAL LEARNER ACTIVITY}

This section focuses on research question R1, i.e., the effects the robot interaction strategy has on the amount of individual learner's verbal activity, together with the main research question R, i.e., if learners with low verbal activity also rate the robot and/or the practice lower post-session.

It should first be noted that the task (improving the learners' verbal communication skills) requires that the learner is participating in the conversation and a low verbal activity is therefore in itself problematic. Low verbal activity may be due either to the level of the conversation being too difficult for the learner (thus hindering task engagement) or that it is not perceived as being interesting (related to social engagement), but in either case an adaptation of the robot's interaction strategy is required to activate or re-engage a disengaged learner [53].

Since the underlying aim of this work is to identify practice sessions that may be suspected to be ineffective for learning, we are more interested in identifying low verbal learner activity 

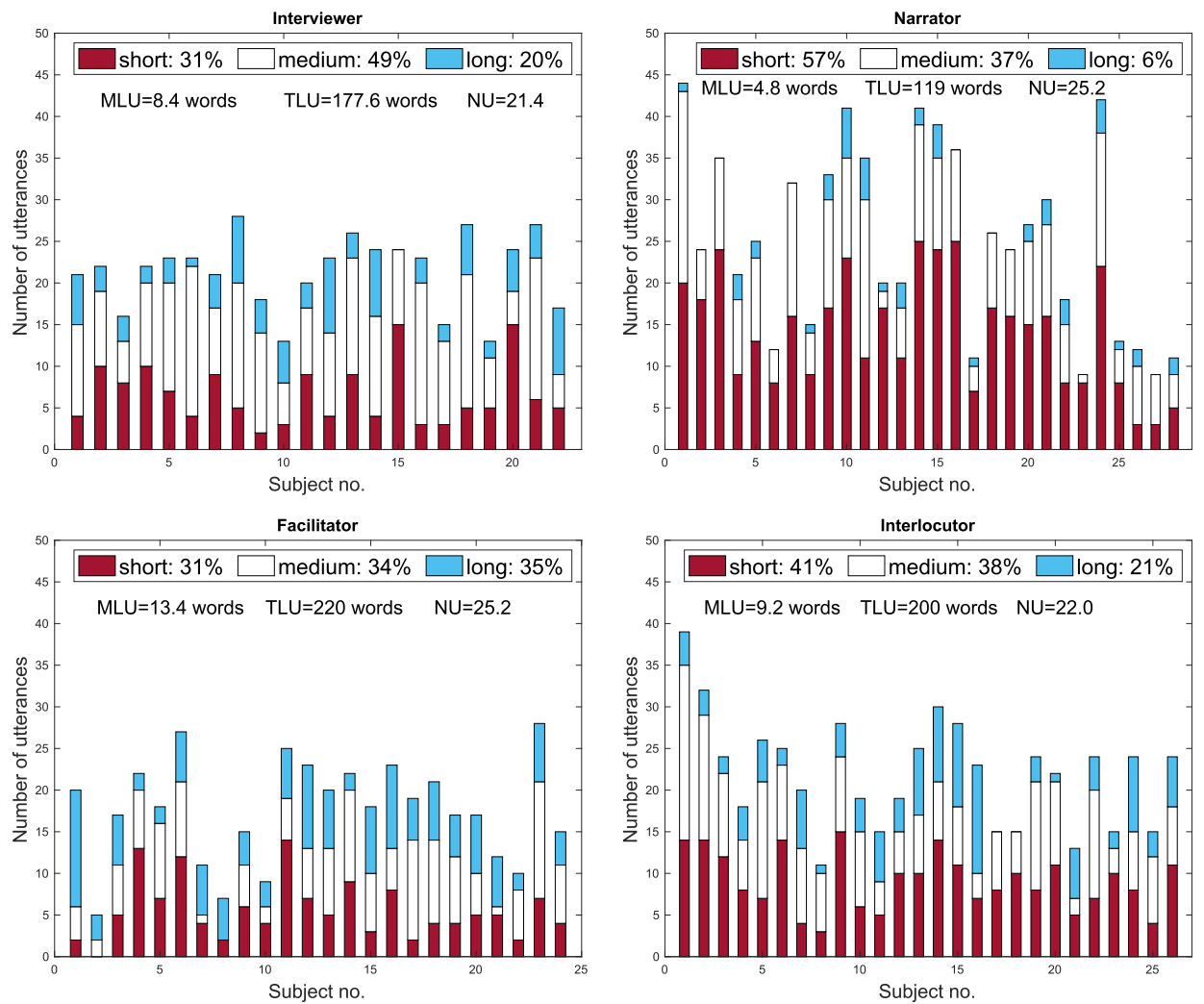

Fig. 3. Total number of learner utterances (NU, bar height), divided into short ( $<4$ words), medium (4-11 words) and long (>11 words) for each subject. The legend states the ratio of utterance lengths, plus the MLU, mean TLU and NU. The subjects have been sorted so that the first 18 subjects are the same in all four graphs, whereas the remaining subject numbers relate to partly different learners.

than the relationship between robot interaction strategy and verbal activity per se. We therefore primarily focus on detecting ineffective spoken L2 conversation practice in the sense that verbal input from the learner becomes scarce or very short, but we have previously found that different robot interaction strategies lead to quantitative and qualitative differences in the learners' verbal activity on the group level [23]. The analyses were performed on the conversation transcriptions, using word count in learner utterances.

\subsection{Statistical Measures for Verbal Learner Activity}

Measures for learner activity included the number of utterances (NU) during a session, the mean length of utterances (MLU), the resulting total length of the learner utterances (TLU), and the ratio of very short utterances (fewer than 4 words, $\phi_{\text {short }}$ ), as shown in Figure 3 . The number of utterances NU indicates how dynamic the interaction is (the higher the number of turns, the more active the exchange between the interlocutors), TLU how much total spoken practice the learner got during the session, and MLU and $\phi_{\text {short }}$ the learner's ability or choice to be verbose (MLU being a standard measure for a language learner's linguistic level).

We first tested if the self-rated learner proficiency level among the 18 subjects who had conversations with all robot interaction strategies influenced the learners' activity. There were no sta- 
tistically significant differences for NU $\left(M_{N U 0}=15.3, n_{0}=4 ; M_{N U 1}=22.0, n_{1}=8 ; M_{N U 2}=24.5, n_{2}=16\right.$; $\left.M_{N U 3}=332.6, n_{3}=44 ; p_{K W}=0.190\right)$, TLU $\left(M_{T L U 0}=419.5, M_{T L U 1}=325.9, M_{T L U 2}=294.8, M_{T L U 3}=280.9\right.$, $\left.p_{K W}=0.092\right)$, or $\Phi_{\text {short }}\left(M_{\Phi 0}=0.28, M_{\Phi 1}=0.33, M_{\Phi 2}=0.42, M_{\Phi 3}=0.40, p_{K W}=0.205\right)$. There was a significant difference for MLU $\left(p_{K W}=0.029\right)$, as conversations with learner proficiency level 0 $\left(M_{M L U 0}=29.6\right)$ had significantly higher MLU than those with proficiency level $2\left(M_{M L U 2}=13.5\right.$, $\left.p_{M W}=0.007<\alpha_{1}\right)$. The difference compared to proficiency level $1\left(M_{M L U 1}=17.5, p_{M W}=0.042>\alpha_{2}\right)$ and $3\left(M_{M L U 3}=20.8, p_{M W}=0.073\right)$ were not significant, nor were the differences between levels 1 and 3 $\left(p_{M W}=0.909\right)$ or between levels 2 and $3\left(p_{M W}=0.070\right)$. Since it was learners with one of the higher proficiency levels who produced the lowest MLU, we judge that differences in verbal activity is not merely an effect of learner proficiency and therefore continue by exploring how the robot's interaction strategies influenced the learners' activity.

\subsection{Number of Utterances}

As shown in Figure 3, the number of learner utterances per conversation (NU, total bar heights) varied substantially between subjects, $7<\mathrm{NU}<44$ (mean $\left.M_{N U}=21.8\right)$ and there were significant differences between robot interaction strategies $\left(p_{K W}=0.036\right)$. Facilitator (mean $\left.M_{N U}=17.5\right)$ led to significantly fewer utterances than Narrator $\left(M_{N U}=25.2, p_{M W}=0.0075<\alpha_{1}\right)$. It also led to fewer utterances than Interviewer $\left(M_{N U}=21.4, p_{M W}=0.0116>\alpha_{2}\right)$ and Interlocutor $\left(M_{N U}=21.9\right.$, $\left.p_{M W}=0.0126>\alpha_{3}\right)$, but the latter two are non-significant. The differences between Interviewer and Interlocutor $\left(p_{M W}=0.779\right)$, between Interviewer and Narrator $\left(p_{M W}=0.286\right)$ and between Interlocutor and Narrator $\left(p_{M W}=0.340\right)$ were also non-significant.

\subsection{Total Utterance Length}

The mean total length of learner utterances TLU varied between subjects, $139<$ TLU $<662$ words (mean $M_{T L U}=293$ ), and differed significantly between the robot interaction strategies $\left(p_{K W}=1.12 *\right.$ $10^{-11}$ ), with the Narrator strategy (mean $M_{T L U}=119$ words) resulting in significantly fewer words than Interviewer $\left(M_{T L U}=178\right.$ words, $\left.p_{M W}=1.11 * 10^{-7}<\alpha_{3}\right)$, Interlocutor $\left(M_{T L U}=200\right.$ words, $\left.p_{M W}=6.29 * 10^{-8}<\alpha_{2}\right)$, and Facilitator $\left(M_{T L U}=220, p_{M W}=4.64 * 10^{-9}<\alpha_{1}\right)$. The differences between Interviewer and Facilitator $\left(p_{M W}=0.0076<\alpha_{5}\right)$ and between Interlocutor and Facilitator $\left(p_{M W}=0.001<\alpha_{4}\right)$ were also significant, while that between Interviewer and Interlocutor $\left(p_{M W}=\right.$ $0.702)$ was not.

\subsection{Mean Utterance Length}

The mean length of learner utterances MLU also differed between subjects, $1.7<$ MLU $<27.3$ (mean $\left.M_{M L U}=8.8\right)$, and significantly between robot strategies $\left(p_{K W}=3.69 * 10^{-5}\right)$, again with Narrator resulting in significantly shorter utterances (mean $M_{M L U}=4.8$ words) than Interviewer $\left(M_{M L U}=6.4\right.$, $\left.p_{M W}=0.003<\alpha_{3}\right)$, Interlocutor $\left(M_{M L U}=9.2, p_{M W}=9.2 * 10^{-4}<\alpha_{2}\right)$ and Facilitator $\left(M_{M L U}=13.4\right.$, $\left.p=5.8 * 10^{-6}<\alpha_{1}\right)$. The differences between Interviewer and Facilitator $\left(p_{M W}=0.002<\alpha_{4}\right)$ and between Interlocutor and Facilitator $\left(p_{M W}=0.005<\alpha_{5}\right)$ were also significant, while that between Interviewer and Interlocutor was non-significant $\left(p_{M W}=0.756\right)$.

\subsection{Ratio of Utterance Lengths}

Since the main interest is to identify individual interactions that are problematic because they do not sufficiently activate the learners throughout the practice, it may be misleading to consider MLU (since few long utterances from an otherwise passive learner give a high MLU) and to some extent also TLU (since this does not indicate how the words were distributed over the conversation). We therefore also considered the ratios of short ( $<4$ words, bottom red stack in Figure 3), medium (4-11 words, middle white stack in Figure 3), and long (>11 words, top blue stack in Figure 3) 
learner utterances. The rationale for analysing the ratios of learner utterance lengths is that if short utterances dominate, the learner is not sufficiently active and the robot needs to encourage longer learner input to increase the speaking practice. The word length category thresholds were set based firstly on MLU in human-human L2 conversations with adults (range 5.86-10.02, mean 7.5 [56]; mean 7.6 [42]), with short and long utterances thus being respectively below and above this range. Secondly, a qualitative analysis of the utterance content indicated that short utterances are typically feedback to the interlocutors' messages, clarification exchanges, or very basic-level utterances; medium utterances are "normal" conversation utterances for exchanges in which all interlocutors are active and long utterances consist of more elaborated descriptions. Thirdly, these word length limits resulted in a reasonably balanced distribution of utterance length categories (overall utterances, $42 \%$ were short, $40 \%$ medium, and $18 \%$ long).

The ratio of short, medium, and long utterances varied greatly between subjects, with $0.10<$ $\phi_{\text {short }}<0.85$ (mean $M_{\phi}=0.41$ ) and significantly between robot interaction strategies (short: $p_{K W}=1.2 * 10^{-7}$ and long: $\left.p_{K W}=1.0 * 10^{-9}\right)$. Narrator $\left(M_{\phi s h o r t}=0.57\right)$ had a significantly higher ratio of short utterances than Interlocutor $\left(M_{\phi s h o r t}=0.42, p_{M W}=3.4 * 10^{-4}<\alpha_{3}\right)$, Interviewer $\left(M_{\phi s h o r t}=0.31, p_{M W}=5.0 * 10^{-6}<\alpha_{2}\right)$, and Facilitator $\left(M_{\phi \text { short }}=0.31, p_{M W}=4.9 * 10^{-7}<\alpha_{1}\right)$. The difference in short utterance ratio between Interlocutor and Facilitator $\left(p_{M W}=0.009<\alpha_{4}\right)$ and Interviewer and Interlocutor $\left(p_{M W}=0.0116<\alpha_{5}\right)$ were also significant, while that between Interviewer and Facilitator $\left(p_{M W}=0.725\right)$ was not. Similarly, Narrator $\left(M_{\phi l o n g}=0.07\right)$ had a significantly lower ratio of long utterances than Interviewer $\left(M_{\phi l o n g}=0.20, p_{M W}=3.9 * 10^{-5}<\alpha_{2}\right)$, Interlocutor $\left(M_{\phi l o n g}=0.22, p_{M W}=1.0 * 10^{-4}<\alpha_{3}\right)$, and Facilitator $\left(M_{\phi l o n g}=0.33, p_{M W}=2.8 * 10^{-8}<\alpha_{1}\right)$. The differences in long utterance ratio between Interlocutor and Facilitator $\left(p_{M W}=0.004<\alpha_{5}\right)$ and between Interviewer and Facilitator $\left(p_{M W}=0.002<\alpha_{4}\right)$ were also significant, while that between Interviewer and Interlocutor $\left(p_{M W}=0.992\right)$ was not.

Hypothesis $\mathrm{H} 1$ that the robot interaction strategy influences the learner activity is hence confirmed, with Narrator resulting in most learner utterance turns, but a significantly higher proportion of them being very short, thus resulting in significantly lower mean and total utterance lengths. Facilitator on the contrary resulted in significantly fewer learner utterances, but with a significantly higher proportion of them being long, thus resulting in a significantly higher mean and total utterance length. These findings are hence in line with the descriptions of the interaction strategies (Section 4.1). Six learner interactions (subjects 6, 8, 17, 23 with Narrator and subjects 7, 10 with Facilitator) have both a low number of utterances and a high ratio of these being short.

\subsection{Relationship between Verbal Activity and Ratings}

We next investigated the main hypothesis $\mathrm{H}$, i.e., that the category of the least verbally active learners were also more negative in their post-session ratings than the other learners. Table 2 reports the threshold values found, the number of learners with a low activity that were detected, and the differences in ratings of learners with low activity compared to other learners. Low active learners rated the robot's Friendliness and Human-likeness significantly lower than more active learners. For the other survey categories, their ratings were often lower, but the differences were not significant.

Hypothesis $\mathrm{H}$ is thus confirmed, i.e., learners with low verbal activity rated the robot significantly lower on the social competency Friendliness and Human-likeness. It may be remarked that the threshold for NU in fact rather identifies that the most active learners were more positive than the other learners. Lower ratings were found also for low thresholds, but they were non-significant (e.g., Conversation $\Delta=-0.21, \mathrm{p}=0.094$ for $\mathrm{NU}=10$, and $\mathrm{n}=5$ ). Given the fact that practice with 
Table 2. Differences in Post-session Ratings between Learners with Low Verbal Activity Compared to Other Subjects, Depending on Measure of Activity (NU: Number of Learner Utterances, TLU: Total Length of Utterances, MLU: Mean Utterance Length, $\phi_{\text {short }}$ : Ratio of Short Utterances to the Total)

\begin{tabular}{l|cccccccc}
\hline & \multicolumn{7}{|c}{$\Delta$ in rating $(\mathrm{p})$} \\
& $\mathrm{NU}=36.1(\mathrm{n}=94)$ & $\mathrm{TLU}=64(\mathrm{n}=8)$ & $\mathrm{MLU}=4.8(\mathrm{n}=17)$ & $\phi_{\text {short }}=0.45(\mathrm{n}=40)$ \\
\hline Learning & -0.21 & $(\mathrm{p}=0.241)$ & --0.05 & $(\mathrm{p}=0.665)$ & 0.10 & $(\mathrm{p}=0.554)$ & 0.08 & $(\mathrm{p}=0.358)$ \\
Friendly & $-\mathbf{0 . 3 0}$ & $(\mathbf{p}=\mathbf{0 . 0 3 7})$ & $-\mathbf{0 . 2 0}$ & $(\mathbf{p}=\mathbf{0 . 0 3 4})$ & $-\mathbf{0 . 3 9}$ & $(\mathbf{p}=\mathbf{0 . 0 1 1})$ & $-\mathbf{0 . 1 6}$ & $(\mathbf{p}=\mathbf{0 . 0 3 3})$ \\
Personal & 0.39 & $(\mathrm{p}=0.571)$ & -0.5 & $(\mathrm{p}=0.672)$ & -0.18 & $(\mathrm{p}=0.351)$ & -0.02 & $(\mathrm{p}=0.548)$ \\
Conversation & -0.15 & $(\mathrm{p}=0.336)$ & 0.46 & $(\mathrm{p}=0.900)$ & -0.10 & $(\mathrm{p}=0.962)$ & -0.11 & $(\mathrm{p}=0.255)$ \\
Human-like & $\mathbf{- 0 . 2 0}$ & $(\mathbf{p}=\mathbf{0 . 0 2 8})$ & 0.06 & $(\mathrm{p}=0.180)$ & -0.03 & $(\mathrm{p}=0.230)$ & -0.05 & $(\mathrm{p}=0.782)$ \\
\hline
\end{tabular}

Numbers in the heading indicate the threshold below which subjects were classified into the low category and the number (n) of subjects below the threshold. Significance level indicated by $p$-values.

low learner activity is less effective for learning in addition to the lower ratings in two of the categories, it is important to adapt the robot strategy when low learner activity is detected.

\section{LEARNER ENGAGEMENT SIGNALLED BY VOCAL FEATURES}

This section continues with research question R2, that is if the robot interaction strategy also influences the learner's emotional engagement, as determined by a human listener (Section 6.1) and a machine learning classification (Section 6.2), together with research question R, i.e., if learners with low emotional engagement levels rate the robot and the practice lower. In addition, research question $\mathrm{R} 4$ is addressed, with respect to the correspondence between manual annotations and automatic classifications (Section 6.3).

All available recordings (one per learner and conversation, in total, 100 audio files) were first annotated by one annotator with respect to the learner's emotional engagement level. An automatic speech detection (the speechDetect function in Matlab's Audio toolbox) was used to detect learner utterances, using a pre-set threshold (empirically determined based on the entire test set) and a periodic Hann window. However, the detected speech segments were manually processed to remove utterances that were from the robot or peer learner, to crop out disturbance noise resulting from touching of the microphone, and to slightly adjust segment boundaries to include full utterances. For the engagement annotation, segments shorter than 1 second were discarded, as annotation of such short segments risks being unreliable. The resulting set of annotated utterances is a sub-set of all utterances, but it is nevertheless adequate for comparisons between robot interaction strategies and between learners.

\subsection{Manually Annotated Vocal Learner Engagement}

The learner utterances were manually annotated as High and Low engagement and Linguistic problems by an annotator with a background in phonetics and speech production research as well as extensive experiences of assessing interactions between language learners and computer-animated virtual teachers or robots. The annotation protocol was inspired by the annotations in [58], where involvement (amusement, disagreement and other) was contrasted with non-involvement based on levels of affect in the speaker's voice. Acoustic features signaling higher affect and engagement are pitch, spectral energy and duration parameters [60] and syllabicity, pitch slope, and loudness [29]. However, syllabicity and duration may not be valid for L2 conversations, since a learner that is interested in the conversation may have linguistic difficulties that reduce speaking rate and introduce pauses. The present annotation therefore aimed at assessing pitch variations and spectral 

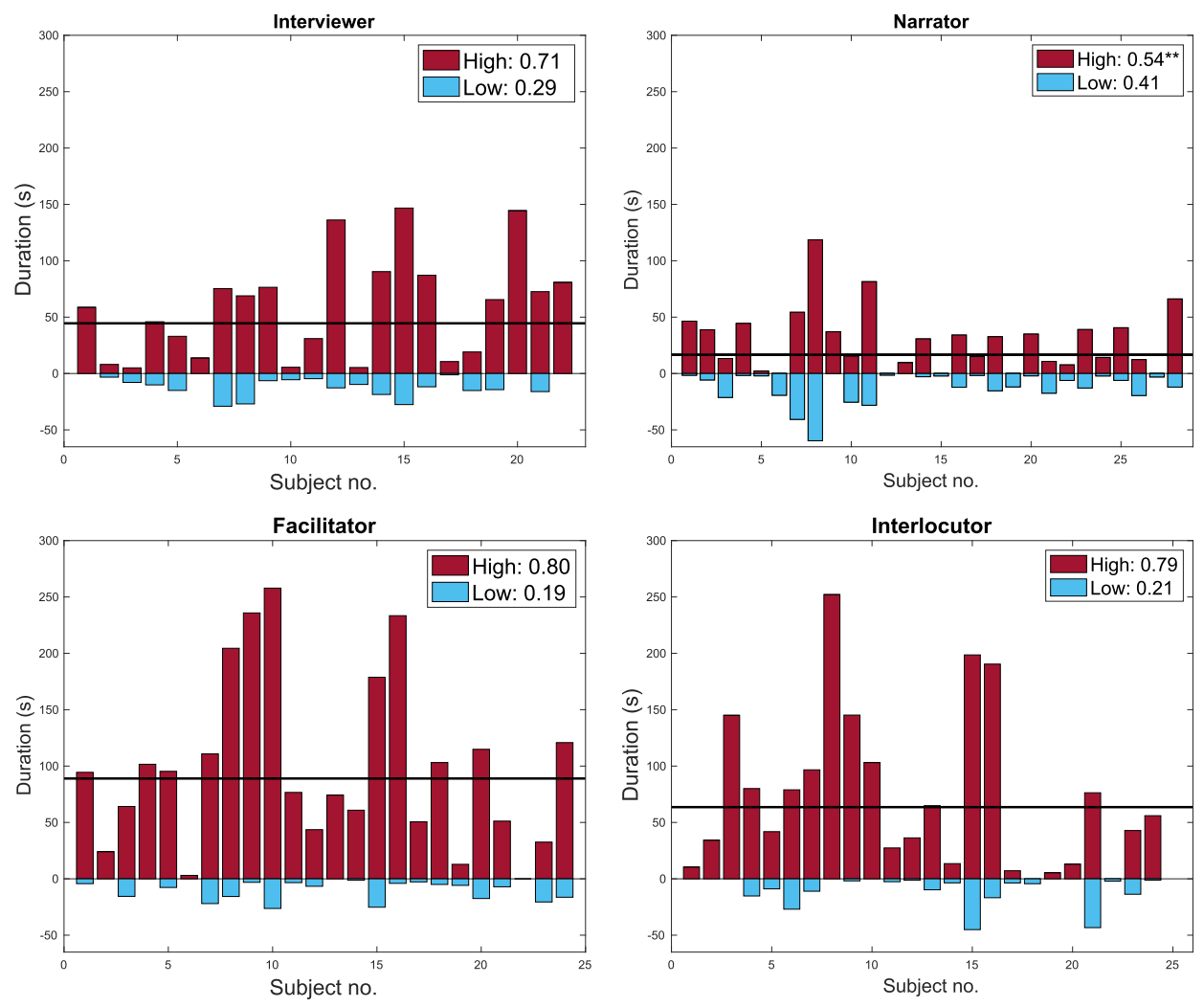

Fig. 4. Total time $(\Sigma)$ and ratio $\left(\phi_{t}\right)$ of learner utterances labelled as having high or low emotional arousal for the different robot strategies and subjects. The horizontal line indicates the level of balance between high and low arousal. The legend figures give the mean ratio of high and low arousal utterances. The subjects have been sorted so that the first 18 subjects are presented in the same order in all four graphs, whereas the remaining subject numbers relate to partly different subjects. ${ }^{* *}$ denotes that the ratio is significantly lower $(p<0.005)$ than Facilitator and Interlocutor using Mann-Whitney U tests.

energy perceptually (no measures were made), regardless of the verbal content or if linguistic difficulties reduced the speaking rate, to determine how interested the learner appeared to be in the spoken exchange. The Linguistic problems category was used for utterances containing additional disturbances, e.g., longer fillers or pauses, which made the annotation of either High and Low engagement inadequate.

The High engagement annotations dominated for most participants, which is probably explained firstly by the conversations being relatively short, and secondly by the experiment being the subjects' first encounters with the social robot, which means that the novelty effect may lead to general high engagement [34].

The measures of engagement for each subject-conversation pair were the total duration of engaged utterances $(\Sigma)$, the ratio of the duration of engaged utterances to the total duration of all utterances $(\Phi t)$, and the ratio of the number of engaged utterances to the total number of utterances $(\Phi n)$. As shown in Figure 4 the duration of engaged utterances differed between subjects (83seconds $<\sum<644$ seconds), between robot interaction strategies (as shown by the High-Low 
Table 3. Differences in Mean Post-session Ratings between Learners Manually Annotated as Low-engaged Compared to Other Learners, Depending on Measure of Engagement

( $\Sigma$ : Duration of Engaged Utterances, $\Phi t$ : Duration Ratio of Engaged Utterances to the Total Duration and $\Phi n$ : Ratio of Number of Engaged Utterances to the Total Number)

\begin{tabular}{l|cccccc}
\hline Survey category & \multicolumn{7}{|c}{$\Delta$ in rating (p-value) } \\
& $\sum=33 \mathrm{~s}(\mathrm{n}=39)$ & $\Phi_{t}=0.4(\mathrm{n}=39)$ & $\Phi_{n}=0.555(\mathrm{n}=17)$ \\
\hline Learning & 0.04 & $(\mathrm{p}=0.434)$ & 0.10 & $(\mathrm{p}=0.494)$ & 0.12 & $(\mathrm{p}=0.579)$ \\
Friendly & $-\mathbf{0 . 1 6}$ & $(\mathbf{p}=\mathbf{0 . 0 1 2})$ & $-\mathbf{0 . 3 0}$ & $(\mathbf{p}=\mathbf{0 . 0 0 7})$ & $-\mathbf{0 . 4 0}$ & $(\mathbf{p}=\mathbf{0 . 0 2 8})$ \\
Personal & 0.04 & $(\mathrm{p}=0.288)$ & -0.27 & $(\mathrm{p}=0.675)$ & -0.17 & $(\mathrm{p}=0.537)$ \\
Conversation & -0.10 & $(\mathrm{p}=0.253)$ & 0.17 & $(\mathrm{p}=0.356)$ & -0.08 & $(\mathrm{p}=0.346)$ \\
Human-like & $-\mathbf{0 . 0 6}$ & $(\mathbf{p}=\mathbf{0 . 0 0 4})$ & 0.26 & $(\mathrm{p}=0.319)$ & -0.01 & $(\mathrm{p}=0.088)$ \\
\hline
\end{tabular}

The heading indicates the thresholds for classifying subjects into the low engagement categories and the number of subjects classified as low engaged.

ratios) and between subject-strategy combinations (shown by the within-subject differences for the first 18 subjects, who are presented in the same order in all four graphs).

As for learner activity, we first checked for significant differences with respect to learner proficiency level, but it did not give rise to any statistically significant differences in length of engaged utterances $\left(p_{K W}=0.22\right)$ or ratio of engaged utterances $\left(p_{K W}=0.19\right)$. We therefore continued by investigating if the differences in engagement duration $(\Sigma)$ or ratio $(\Phi t, \Phi n)$ were instead related to the robot interaction strategy. It was found that the differences in engagement duration $\left(p_{K W}=0.0023\right)$, engagement time ratio $\left(p_{K W}=0.0011\right)$, and engaged turn ratio $\left(p_{K W}=0.0013\right)$ were statistically significant. Narrator $\left(M_{\Sigma}=28.8 \mathrm{~s}\right)$ led to significantly shorter duration of engaged utterances than Facilitator $\left(M_{\Sigma}=95.6 \mathrm{~s}, p_{M W}=2.16 * 10^{-4}<\alpha_{1}\right)$, Interlocutor $\left(M_{\Sigma}=\right.$ $\left.71.2 \mathrm{~s}, p_{M W}=0.0056<\alpha_{2}\right)$ and Interviewer $\left(M_{\Sigma}=55.9 \mathrm{~s}, p_{M W}=0.0161<\alpha_{3}\right)$. Furthermore Narrator $\left(M_{\Phi t}=0.5, M_{\Phi n}=0.54\right)$ led to a significantly lower engaged time ratio than Interlocutor $\left(M_{\Phi t}=0.82, p_{M W}=0.0014<\alpha_{2}\right)$ and Facilitator $\left(M_{\Phi t}=0.86, p_{M W}=6.3 * 10^{-4}<\alpha_{1}\right)$ and to a significantly lower engaged turn ratio than Interlocutor $\left(M_{\Phi n}=0.79, p_{M W}=9.6 * 10^{-4}<\alpha_{2}\right)$ and Facilitator $\left(M_{\Phi n}=0.81, p_{M W}=6.4 * 10^{-4}<\alpha_{1}\right)$. The other differences between Narrator and Interviewer $\left(M_{\Phi t}=0.77, p_{M W}=0.135 ; M_{\Phi n}=0.71 p_{M W}=0.0750\right)$, between Interviewer and Facilitator $\left(p_{M W \Phi t}=0.0127>\alpha_{3}, p_{M W \Phi n}=0.0436>\alpha_{4}\right)$, between Interviewer and Interlocutor (respectively, $p_{M W \Sigma}=0.584, p_{M W \Phi t}=0.0751, p_{M W \Phi n}=0.0491>\alpha_{5}$ ), Interviewer and Facilitator (respectively, $p_{M W \Sigma}=0.0925, p_{M W \Phi t}=0.0127>\alpha_{3}, p_{M W \Phi n}=0.0436>\alpha_{4}$ ), and Interlocutor and Facilitator (respectively, $p_{M W \Sigma}=0.24, p_{M W \Phi t}=0.928, p_{M W \Phi n}=0.875$ ) were not significant.

Hypothesis $\mathrm{H} 2$ is hence confirmed, with Facilitator and Interlocutor resulting in the highest ratio of utterances annotated as engaged and Narrator in the lowest.

Continuing with hypothesis $\mathrm{H}$, the relationships between the post-session ratings and the total duration, time and turn ratios of engaged utterances $(\Sigma, \Phi t, \Phi n)$ were next investigated. As for learner activity (Section 5.1), the hypothesis is that learners below given thresholds of engagement rated the robot lower than the other learners, and guided by the distribution shown in Figure 4 different threshold values were searched.

As shown in Table 3, learners who were below the low engagement thresholds $(\Sigma=33 \mathrm{~s}$, $\Phi t$ $=0.4, \Phi n=0.555)$ rated the robot significantly lower regarding how Friendly and Human-like it was. Hypothesis $\mathrm{H}$ is thus confirmed for manual classification on vocal emotional features, with significant differences regarding two social competencies of the robot (Friendliness and Humanlikeness). 

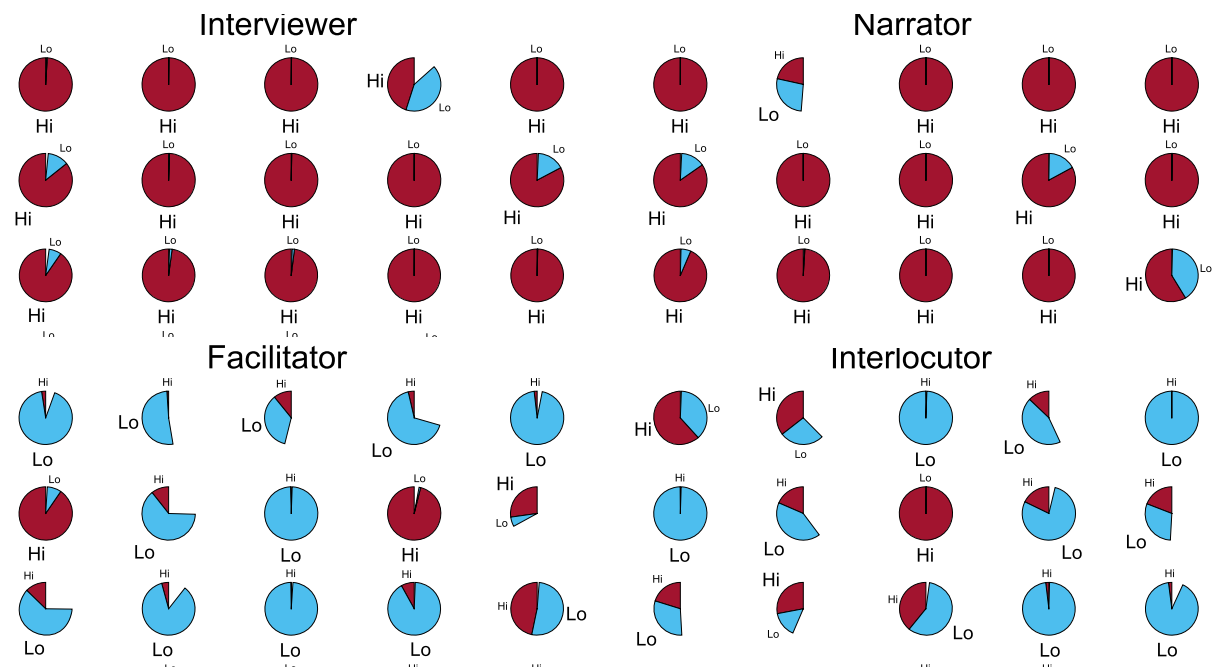

Interlocutor
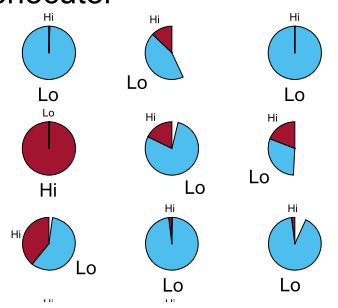

Fig. 5. Probabilities for high (Hi, dark red), low (Lo, light blue), and neutral (missing part of the pie) emotional arousal in the first 15 utterances by one subject in conversations with the four different robot strategies.

\subsection{Learner Engagement Classification Through Automatic Detection of Vocal Arousal}

We next maintained the focus on research questions $\mathrm{R}$ and $\mathrm{R} 2$ regarding if the robot interaction strategy influenced the learners' vocal signals of emotional engagement, and the relation to the post-session ratings, but now in conjunction with research question R5, i.e., if a standard state-ofthe-art method can be used to analyse the emotional engagement with similar classification results as with manual annotation in Section 6.1.

For the automatic detection, the bidirectional Long Short Time Memory (LSTM) network Speech Emotion Recognition implementation in the Matlab Deep Learning Toolbox was used. It trains a network to recognise the seven emotions anger, happiness, boredom, sadness, neutral, disgust, and anxiety/fear, based on a database of 535 sentences, in which 10 actors read emotionindependent German sentences with the different emotions [10].

The present experiment employed the standard training of the network, which means that the network is trained to give the probabilities for the different emotions based on acoustic features extracted using a $600 \mathrm{~ms}$ windows with $300 \mathrm{~ms}$ overlap. A sequential feature selection [33] is used to incrementally determine the feature set that results in the highest accuracy. In the present study, the output of the biLSTM was then mapped onto three arousal level classes High, Neutral, or Low, based on the emotion's value along the Arousal/Active-Passive dimension. That is, anger and happiness were mapped onto the High arousal category, neutral to Neutral, and boredom and sadness to Low arousal, while anxiety and disgust were discarded as non-relevant in this setting. The output of the biLSTM model is in the form of the probability of each of the three levels of arousal, as illustrated in Figure 5. Each utterance was then labelled with the dominating arousal level, which we stipulate is related to the learner's level of emotional engagement for that utterance.

Figure 5 shows substantial differences in learner arousal between conversations with different robot strategies for the same learner during the same portion of the conversation. Since other factors, such as which peer learner the conversation was with and the topics discussed during the conversation, may also influence the arousal level of an individual participant, Figure 5 is an illustration of how the arousal level may vary between conversations rather than an indication of 

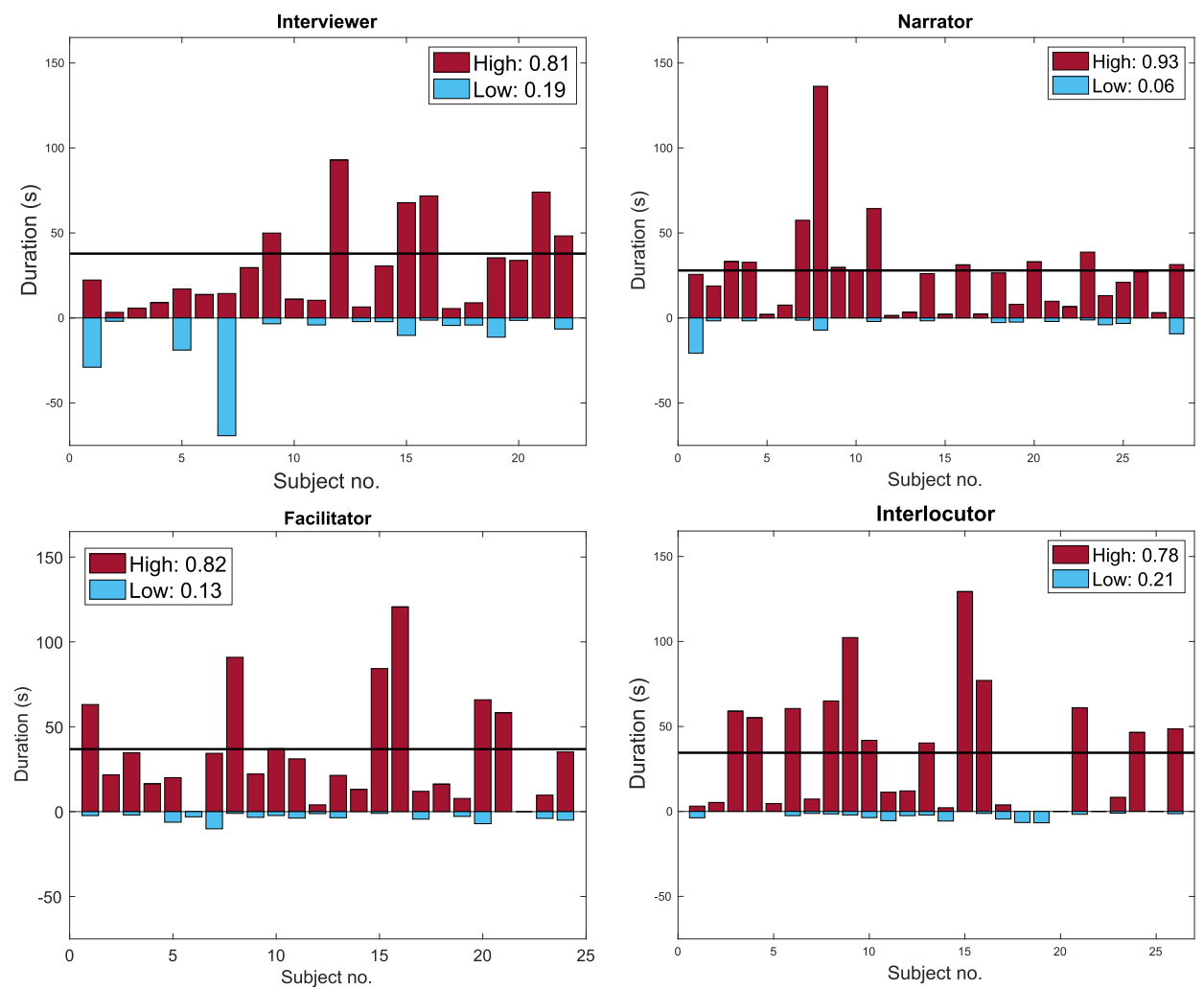

Fig. 6. Total duration (bar height) and ratio (difference between positive and negative bars) of learner utterances detected as having high or low emotional arousal for the different robot strategies and subjects. The horizontal line indicates the level of balance between high and low arousal. The legend figures give the ratio of high and low arousal utterances. The subjects have been sorted so that the first 18 subjects are presented in the same order in all four graphs, whereas the remaining subject numbers relate to partly different subjects.

differences between robot interaction strategies. Over the entire conversation, Figure 6 illustrates that the distribution of high vs. low arousal differed between learners (e.g., subject 6 has almost no utterances with Low arousal and subject 10 has more Low than High arousal utterances regardless of robot strategy) and between robot strategies. The measures considered were the duration of High arousal utterances $(\Sigma)$, the ratio of the duration of High arousal utterances to the total duration $(\Phi t)$, and the ratio of the number of High arousal utterances to the total number $(\Phi n)$. None of the differences were significant $\left(p_{K W \Sigma}=0.685, p_{K W \Phi t}=0.123, p_{K W \Phi n}=0.098\right)$. Narrator had the highest time and turn ratios $\left(M_{\Phi t}=0.93, M_{\Phi n}=0.87\right)$, but the shortest duration $\left(M_{\Sigma}=25.6\right)$. Facilitator, on the contrary, had the longest duration $\left(M_{\Sigma}=34.7\right)$, the lowest turn ratio $\left(M_{\Phi n}=0.71\right)$ and the second highest time ratio $\left(M_{\Phi t}=0.88\right)$. Interviewer $\left(M_{\Sigma}=30.1, M_{\Phi t}=\right.$ $\left.0.81, M_{\Phi t}=0.77\right)$ resulted in slightly more High arousal than Interlocutor $\left(M_{\Sigma}=32.5, M_{\Phi t}=0.79\right.$, $\left.M_{\Phi t}=0.72\right)$, regardless of measure.

Hypothesis $\mathrm{H} 2$ is hence not confirmed by the automatic labeling of arousal, in contrast to the manual annotations of engagement in Section 6.1, and no direct mapping between automatic High arousal labels and manual high engagement labels is hence demonstrated. One explanation for this may be that whereas the engagement annotation in Section 6.1 disregarded hesitations that lower the speech rate and the signal power spectrum over the utterance, such factors influence the 
Table 4. Differences in Mean Post-session Ratings between Learners Automatically Detected as Having Lower Arousal Compared to Other Learners, Depending on Measure of Engagement ( $\Sigma$ : Duration of High Arousal Utterances, $\Phi_{t}$ : Duration Ratio of High Arousal Utterances to the Total Duration, and $\Phi_{n}$ : Ratio of Number of High Arousal Utterances to the Total Number)

\begin{tabular}{l|cccccc}
\hline Survey category & \multicolumn{7}{|c}{$\Delta$ in rating $(\mathrm{p}-\mathrm{value})$} \\
& \multicolumn{2}{|c}{$5.5 \mathrm{~s}(\mathrm{n}=21)$} & $\phi_{t}=0.44(\mathrm{n}=5)$ & $\phi_{n}=0.44(\mathrm{n}=10)$ \\
\hline Learning & -0.03 & $(\mathrm{p}=0.502)$ & $-\mathbf{0 . 1 1}$ & $(\mathbf{p}=\mathbf{0 . 0 0 8})$ & -0.11 & $(\mathrm{p}=0.232)$ \\
Friendly & -0.47 & $(\mathrm{p}=0.161)$ & 0.20 & $(\mathrm{p}=0.107)$ & -0.21 & $(\mathrm{p}=0.246)$ \\
Personal & -0.23 & $(\mathrm{p}=0.076)$ & -0.76 & $(\mathrm{p}=0.429)$ & $-\mathbf{0 . 3 6}$ & $(\mathbf{p}=\mathbf{0 . 0 2 7})$ \\
Conversation & $\mathbf{- 0 . 1 8}$ & $(\mathbf{p}=\mathbf{0 . 0 3 2})$ & -0.26 & $(\mathrm{p}=0.220)$ & 0.34 & $(\mathrm{p}=0.415)$ \\
Human-like & $\mathbf{- 0 . 1 3}$ & $(\mathbf{p}=\mathbf{0 . 0 2 7})$ & -0.23 & $(\mathrm{p}=0.161)$ & 0.28 & $(\mathrm{p}=0.440)$ \\
\hline
\end{tabular}

The heading indicates the thresholds for classifying subjects into the low engagement categories and the number of low arousal subjects.

detection of arousal. In order to investigate the extent to which they do measure similar emotional states, we investigate the correspondence between the labels in Section 6.3.

However, we first revisit Hypothesis $\mathrm{H}$ by investigating if learners with a low duration $(\Sigma)$ or a low ratio $\left(\Phi_{t}, \Phi_{n}\right)$ of High arousal utterances rated the robot lower. Following the same procedure as in Section 6.1, thresholds for low levels of High arousal were searched, guided by the levels observed in Figure 6. Significant differences were found for all ratings except Friendliness, as shown in Table 4.

Hypothesis $\mathrm{H}$ is thus confirmed in that learners with a lower level of High arousal rated Learning $\left(\phi_{t}\right)$ and the robot's Personal $\left(\phi_{n}\right)$, Conversation $(\Sigma)$, and Human-likeness skills $(\Sigma)$ significantly lower.

\subsection{Relationship between Manual Engagement Annotations and Automatically Detected Arousal}

The previous section showed that automatic labeling of arousal levels detect categories of learners with low arousal that differ in their post-session ratings of the practice and the robot. This confirms that automatic detection of arousal can be an important tool in determining if learners have a low engagement. However, as stated in hypothesis H5, it is further desirable that the automatic detection make similar judgements as a human observer, and the relationship between the manual annotation of engagement in Section 6.1 and the automatic labeling of arousal in Section 6.2 was therefore analysed.

Using the duration ratio $\phi t$, the automatic labels of arousal level were compared with the manual annotation of engagement for individual learners in each conversation. The ratios of engagement and arousal were calculated for every learner-conversation combination, and the correspondence between the two ratios was calculated in terms of classifications into low or high arousal/engagement, as shown in Figure 7. A learner was considered as belonging to the respective low arousal and low engagement categories if the ratio of utterances with high arousal/engagement was below a threshold and to the respective high categories if the ratio was above. The threshold was varied and the matches in low and high classifications were then counted, as exemplified in Figure 7. It should be noted that these numbers do not constitute a confusion matrix, since the labels and the objective of the annotation were different, but if the distribution is used to calculate would-be "precision" (0.51-0.81) and "recall" $(0.51-0.82)$ for the majority class at different thresholds and the resulting "balanced accuracy" $(0.56-0.62)$, the correspondence is well above chance level. Considering the difference in annotation task, in addition to the differences in 

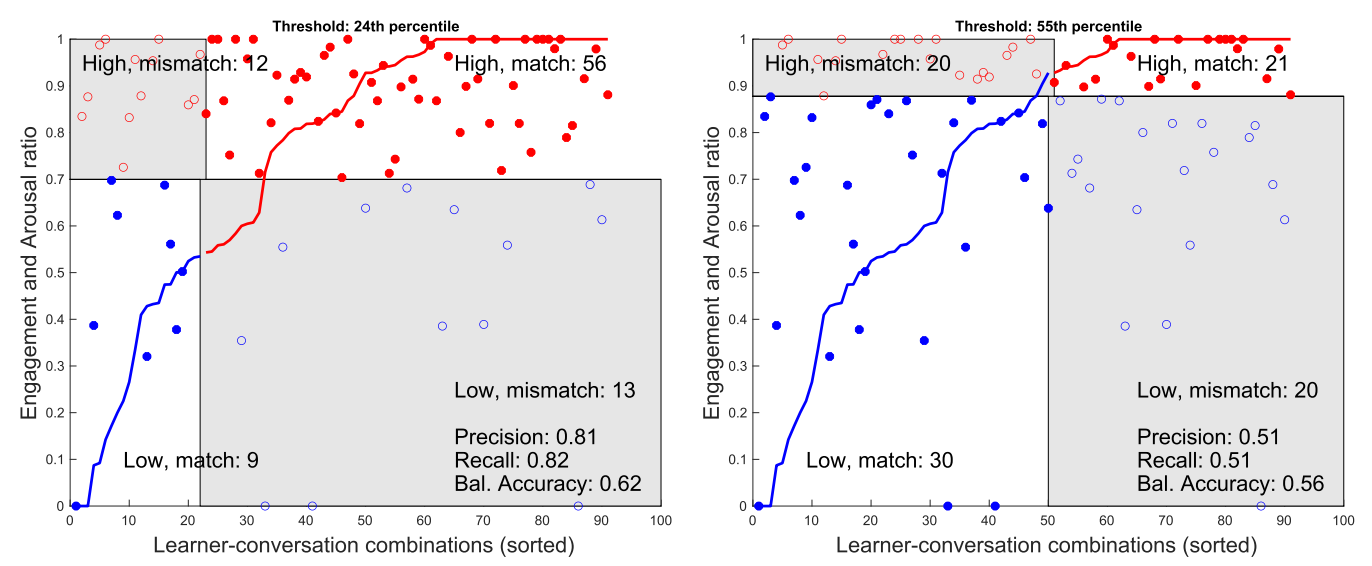

Fig. 7. Correspondence between the automatic method (solid blue and red line) and manual annotation (filled and unfilled circles) regarding classification of learners with low and high arousal/engagement. Classification is made on the conversation level (i.e., each circle, and each data point on the solid line, represents one learner in one conversation). One low and one high classification threshold are shown. Lower left area (with filled blue circles and blue line) and upper right area (with filled red circles and red line) show learners that both methods classify as, respectively, low- and high-engaged, and the two shaded areas (with unfilled circles) indicate a mismatch in classification.

the recordings (conversational vs. read speech, acted $v s$. natural emotions/arousal, L1 vs. L2 speakers, German vs. Swedish, high-quality lab recordings vs. recordings in a real setting), the arousal detection can be considered a promising method to detect engagement, in particular since previous work [55] has shown low performance $(25 \%-53 \%)$ for training and test sets from different sources.

Hypothesis $\mathrm{H} 5$ is hence confirmed, in that the correspondence between manual annotations and automatic labels, measured through classification similarity, is at a reasonably high level.

Both the manual annotations of engagement and the automatic detection of learner arousal showed that groups of learners with lower engagement can be detected and that these learners rate the practice and/or the robot lower along certain dimensions. As analyses based on spoken input are restricted to when a learner is active, it is of interest to investigate visual signals since they may be applied also when the learner is thinking about answering or is listening to the robot or peer, as described next.

\section{LEARNER ENGAGEMENT SIGNALLED BY FACIAL EXPRESSIONS}

Facial expressions and gaze provide important cues to determine learners' affective state, if they follow or are confused, and if they are engaged or not in the practice. Two different sets of experiments are therefore performed: Firstly a general affective analysis of participants (Section 7.1) and secondly an engagement analysis specifically focused on the non-active participant (Section 7.2). The objective of the experiments in this section is to investigate research questions R, R4, and R5, i.e., if low-engaged learners who are identified through facial expressions rate the robot and the practice lower (R), if this can be extended to listeners (R4), and if automatic classification of lowengaged learners agree with manual annotations (R5). In addition, research question R3, i.e., if the robot interaction strategy influences the learners' facial emotional expressions, is also investigated, but due to the vast amount of video data and the extremely time-consuming task of annotating 

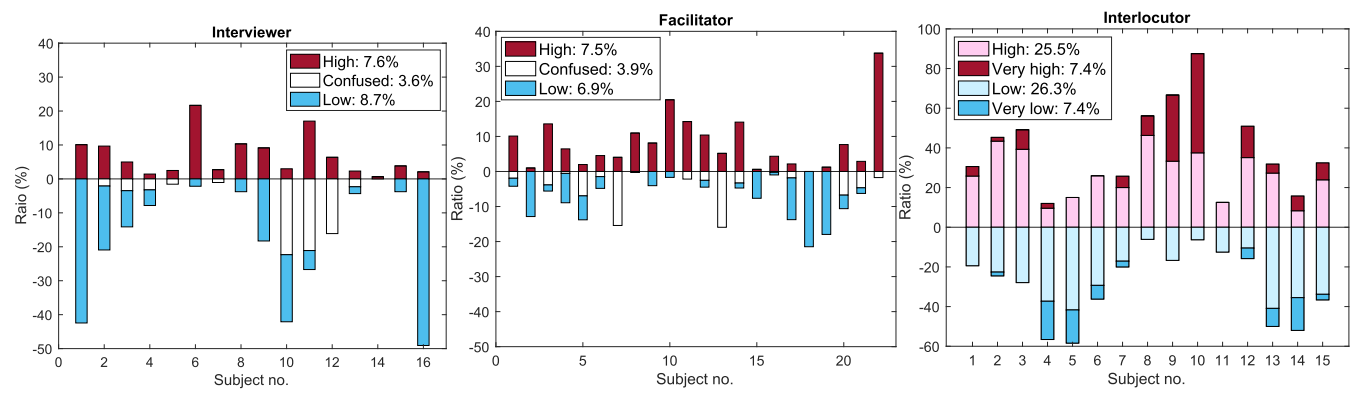

Fig. 8. The ratio of learner/listener sequences annotated as having high and low emotional engagement and (for Interviewer and Facilitator) containing confusion per subjects. The graph for Interlocutor shows the annotations of listener engagement, described in Section 7.2, which used different labels. The legend figures give the ratios over all subjects (the scale is therefore further different). N.B. The subjects have been sorted so that the first 9 subjects are the same in all graphs and the 12 first are the same for the Interviewer and Facilitator. The remaining subject numbers relate to partly different subjects.

video sequences, we performed the experiments in this section on two subsets of the entire dataset: interactions with the Interviewer and Facilitator interaction strategies in Section 7.1 and with the Interlocutor strategy in Section 7.2.

\subsection{Manually Annotated Learner Engagement Using Facial Expressions}

Conversations with 26 learners interacting with the Interviewer and Facilitator interaction strategies were chosen randomly, resulting in 38 video recordings of in total 589,559 frames. These strategies correspond to the largest differences in robot-learner interaction regarding initiative (robot $v s$. learners) and learner involvement (one vs. two learners simultaneously). They were further found to be quite different in terms of engagement signalled through verbal features (Section 5) and are therefore a relevant contrasting pair. Narrator was found to be even more different from Facilitator in some aspects, but was not selected since the robot addressed both learners simultaneously and the strategy; therefore, contained few turn distributions between learners.

The videos, without sound, were manually annotated on the frame level by one annotator each, using four affective classes, defined prior to the annotation task as follows:

High engagement: Display of interest, happiness, delight, and humor, through, e.g., laughter, open-mouth smiles.

Neutral: Display of calm, content and thoughtfulness, with a neutral and/or face and quick, small smiles.

Confusion: Displays of uncertainty through raised eyebrows with a crease between and wrinkled nose.

Low engagement: Display of boredom and loss of interest, signalled, e.g., by looking down, gazing away from the robot, and peer or signals of displeasure with the mouth or the eyebrows.

Three different annotators were used, after a test set consisting of three 5-minute videos resulted in an inter-annotator agreement of 0.9. The distribution of the annotations was heavily unbalanced, with High engagement: 7.4\%, Neutral: $81.6 \%$, Confused: 3.7\%, and Low engagement: $7.3 \%$. The difference in distribution compared to voice-based engagement annotations in Section 6, which had a high ratio of engaged utterances, is explained by the fact that the video-based annotation labels all frames during the entire conversation, whereas the voice-based labels are for the portion when the learner is speaking and disregards sequences of hesitation. 
Table 5. Differences in Mean Post-session Ratings between Learners Below the Thresholds for Facially Displayed Low Engagement and Other Learners, Depending on Measure of Engagement $\left(\phi_{H}\right.$ : Ratio of High

Engaged Video Sequences to the Total, $\phi_{L}$ : Ratio of Low Engaged Video Sequences to the Total and $\delta$ : Balance in Ratio between High and Low Engaged Sequences, i.e., $\phi_{H}-\phi_{L}$ )

\begin{tabular}{l|ccccccccc}
\hline Survey category & \multicolumn{7}{|c}{$\Delta$ in rating (p-value) } \\
& $\phi_{H}=1 \%(\mathrm{n}=3)$ & $\phi_{L}=7.7 \%(\mathrm{n}=11)$ & $\delta=+4.1 \%(\mathrm{n}=25)$ & $\delta=+1.2 \%(\mathrm{n}=18)$ \\
\hline Learning & -1.42 & $(\mathrm{p}=0.126)$ & -0.19 & $(\mathrm{p}=0.744)$ & $-\mathbf{1 . 0 4}$ & $(\mathbf{p}=\mathbf{0 . 0 0 5})$ & -0.57 & $(\mathrm{p}=0.154)$ \\
Friendly & -0.04 & $(\mathrm{p}=0.863)$ & -0.39 & $(\mathrm{p}=0.220)$ & -0.21 & $(\mathrm{p}=0.246)$ & -0.02 & $(\mathrm{p}=0.769)$ \\
Personal & -2.33 & $(\mathrm{p}=\mathbf{0 . 0 1 1})$ & -0.38 & $(\mathrm{p}=0.570)$ & -0.75 & $(\mathrm{p}=0.0904)$ & $-\mathbf{1 . 0}$ & $(\mathrm{p}=\mathbf{0 . 0 1 1})$ \\
Conversation & -1.3 & $(\mathrm{p}=0.157)$ & -0.22 & $(\mathrm{p}=0.632)$ & -0.11 & $(\mathrm{p}=0.837)$ & -0.61 & $(\mathrm{p}=0.171)$ \\
Human-like & -0.77 & $(\mathrm{p}=0.470)$ & $\mathbf{- 1 . 0}$ & $(\mathbf{p}=\mathbf{0 . 0 4 2})$ & -0.55 & $(\mathrm{p}=0.212)$ & $-\mathbf{0 . 9 3}$ & $(\mathrm{p}=\mathbf{0 . 0 2 7})$ \\
\hline
\end{tabular}

The heading indicates the thresholds for classifying subjects into the low engagement categories and the number of subjects classified as having low arousal.

We first compare the engagement levels between the robot interaction strategies, shown in Figure 8 (graphs 1 and 2). There are substantial differences between subjects, but also, smaller, between the robot interaction strategies, with a lower ratio of low engagement for the Facilitator strategy. A Mann-Whitney U-test for the subjects who experienced both settings indicates that the difference regarding the balance in the ratio of High and Low engagement for Interviewer $\left(M_{\delta}\right.$ $=-0.059)$ and Facilitator $\left(M_{\delta}=0.015\right)$ is significant $\left(p_{M W}=0.0459, \mathrm{n}=12\right)$, similar to the results from manual annotations of voice quality (Section 6.1).

We next investigate if the engagement levels that were displayed by the learners' facial actions influenced their post-session ratings. Three different measures were considered, the ratio of high engaged video sequences to the total number of sequences $\left(\phi_{H}\right)$, the ratio of low engaged video sequences to the total number $\left(\phi_{L}\right)$, and the balance between the high and low engaged ratios $(\delta=$ $\left.\phi_{H}-\phi_{L}\right)$. As shown in Table 5, learners below the low engagement threshold rated several aspects of the robot significantly lower than other learners. Which aspects depended on the measure: Personal for $\Phi_{H}$ or $\delta$, Human-like for $\Phi_{L}$ and $\delta$ and Learning for $\delta$. It also depended on the threshold for $\delta$, as exemplified by the differences resulting from setting the threshold to $+4.1 \%$ or $+1.2 \%$.

Hypothesis $\mathrm{H}$ is hence confirmed, since ratings in all post-survey categories were consistently lower for learners with low engagement and the differences were significant in three out of five categories with the measures used.

\subsection{Automatic Classification of Listener Engagement Based on Facial Expressions}

Previous work on robot-assisted language learning studies with adults has been with one learner and one robot [32] or with one learner and two robots [37]. The present setup with two learners is substantially different from if one learner interacted alone with one or two robots, since learners may have a listening role during several dialogue turns between the robot and the other learner, which may cause them to become disengaged. The main advantage of adding video-based analysis of facial expressions for the three-party setting is hence that it can be applied to sequences of the interaction when the learner is not active. In this section, we therefore specifically address research questions, R4, i.e., if listeners' level of engagement is reflected in their facial expressions; R, i.e., if the observed level of engagement is related to their ratings of the practice and the robot and R5, i.e., if manual and automatic classification of engagement agree.

The Interlocutor conversations were used, since the strategy has clearly defined attention shifts between the learners, as opposed to Facilitator and Narrator, and since the shifts were more frequent than with the Interviewer. These conversations are hence the clearest example of the learner acting both as an active and listening participant. 

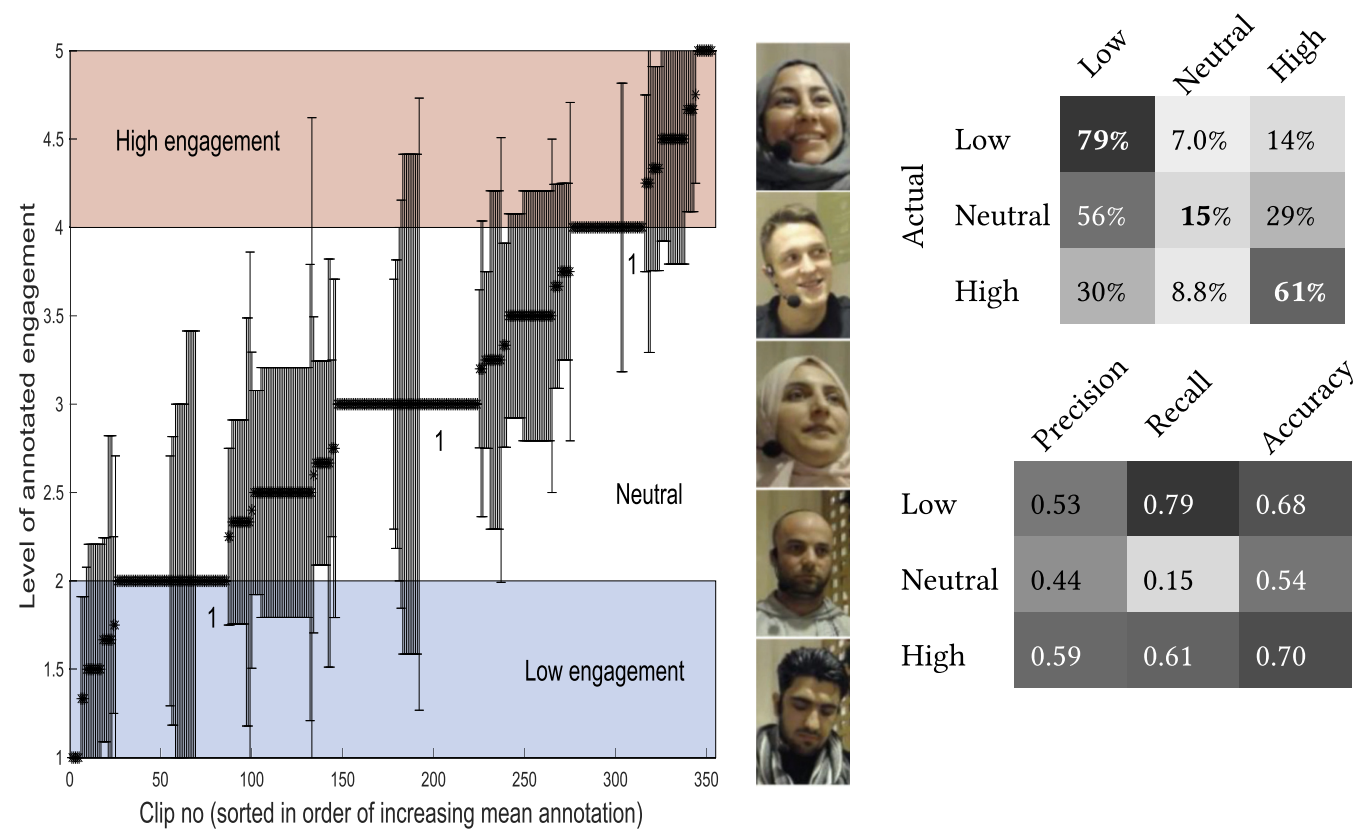

Fig. 9. Left: Mean annotated engagement levels and standard deviation for the 801 video clips sorted in ascending order of engagement. Blue and red background indicate Low and High engagement. Clips marked with 1 were labelled by one annotator. Middle: Photos exemplify, from bottom to top, annotations of "Very disengaged" (1), "Disengaged" (2), "Neutral" (3), "Engaged" (4), and "Very engaged" (5). Right: Confusion matrix (top) and statistics (bottom) for the engagement classification using combined SVMs. Accuracy is the balanced measure using the average of the recall on each class [47].

15 videos of conversations with the Interlocutor strategy were cut into segments of 5 seconds, after empirical testing of appropriate segment lengths for manual annotation of engagement. Segments containing the listener were identified, resulting in 353 video clips. The video clips were annotated as "Very disengaged", "Disengaged", "Neutral", "Engaged", and "Very engaged" by nine volunteers, who were presented a subset of clips in random order in a webinterface with muted video.

In total, 802 annotations were collected, with 62 of the video clips classified by one annotator, 175 by two, 85 by three, 25 by four, and 7 by five. To be able to handle different labels by different annotators, the labels were translated to numerical values from 1 ("Very disengaged") to 5 ("Very engaged"). In order to achieve a more balanced distribution for the classification, the categories were clustered into Low, Neutral, and High and the label of each clip was set according to the mean value, i.e., Low engagement for mean values up to 2, Neutral for means between 2 and 4 and High for mean values 4 and above, as illustrated in Figure 9. For the classification experiments, the video clips for which OpenFace 2.0 [6] could extract FAUs, i.e., Facial Action Units, during at least 4 seconds (120 images) were maintained. This resulted in 801 annotations of engagement, with 59 very disengaged (7.4\%), 211 disengaged (26.3\%), 268 neutral (33.4\%), 204 engaged (25.5\%), and 59 very engaged (7.4\%). Compared to the distribution in Section 7.1, the neutral category is hence smaller and the engaged and disengaged categories larger. It should be noted firstly that the annotators in Section 7.1 only had one category each corresponding to high and low engagement, which may have led to a larger variability in engagement level in the neutral category and secondly 
Table 6. Differences in Mean Post-session Ratings between Listeners Below the Thresholds for Facially Displayed Low Engagement and Other Listeners, Depending on Measure of Engagement (M: Mean for Engagement Labels for the Listener, $\phi_{L}$ : Ratio of Low Engaged Video Sequences to the Total and $\phi_{H}$ : Ratio of Low Engaged Video Sequences to the Total

\begin{tabular}{l|cccccc}
\hline Survey category & \multicolumn{7}{|c}{$\Delta$ in rating $(\mathrm{p}$-value $)$} \\
& \multicolumn{7}{|c}{$\mathrm{M}(\mathrm{n}=5)$} & $\phi_{L}=37 \%(\mathrm{n}=4)$ & $\phi_{H}=25 \%(\mathrm{n}=4)$ \\
\hline Learning & $\mathbf{- 1 . 8}$ & $(\mathbf{p}=\mathbf{0 . 0 2 0})$ & $-\mathbf{2 . 0}$ & $(\mathbf{p}=\mathbf{0 . 0 0 8 8})$ & -1.0 & $(\mathrm{p}=0.154)$ \\
Friendly & $-\mathbf{1 . 3}$ & $(\mathbf{p}=\mathbf{0 . 0 2 3})$ & -1.3 & $(\mathrm{p}=0.0513)$ & -0.98 & $(\mathrm{p}=0.256)$ \\
Personal & -0.5 & $(\mathrm{p}=0.444)$ & -0.38 & $(\mathrm{p}=0.674)$ & -0.05 & $(\mathrm{p}=1.00)$ \\
Conversation & -0.9 & $(\mathrm{p}=0.084)$ & $-\mathbf{1 . 4}$ & $(\mathbf{p}=\mathbf{0 . 0 2 2})$ & -0.68 & $(\mathrm{p}=0.256)$ \\
Human-like & $-\mathbf{1 . 7}$ & $(\mathbf{p}=\mathbf{0 . 0 2 3})$ & $\mathbf{- 1 . 9}$ & $(\mathbf{p}=\mathbf{0 . 0 3 0 8})$ & -1.6 & $(\mathrm{p}=0.185)$ \\
\hline
\end{tabular}

The heading indicates the thresholds for classifying subjects into the low engagement categories and the number of subjects with low engagement.

that the labels in Section 7.1 are for the entire conversation, whereas the ones here are specifically for turns when the robot and the peer are interacting.

The inter-rater agreement of the human annotators for the three categories was calculated using Fleich $\kappa$ [30], to be $\kappa=0.73$, which is a level considered to be a "substantial agreement" [39]. Disagreement between raters occurred in particular when the listener diverted the gaze, which some annotators interpreted as concentrated listening, while others as disinterest. The mean engagement level per subject over the conversation was Neutral for 11 listeners, High for four and Low for none.

We next revisited the main research question R, i.e., if the observed learner engagement levels are reflected in their post-session ratings. As shown in Table 6, listeners in the Low category rated the practice and robot significantly lower on all dimensions except how Personal the robot was.

Hypothesis $\mathrm{H}$ is hence again confirmed, in that low-engaged listeners rated the robot and the practice significantly lower than other listeners in four out of five post-session survey categories.

We next focused on research question R5, i.e., if automatic classification of listener engagement agree with manual annotations. FAU and vertical gaze direction were extracted using OpenFace 2.0 [6]. After preliminary experiments, 17 FAU (AU01-02, 04-07, 09-10, 12, 14, 15, 17, 20, 23, 25, 26, $45)$ were selected empirically. These FAU relate to movements of the eyebrows (raising and lowering), lids (blinking, raising and tightening), lips (raising, corner pulling and depressing, stretching, tightening, parting), cheek raiser, nose wrinkler chin, and jaw (drop).

The classification was performed using a combination of two SVM with, respectively, a linear and a radial basis function (RBF) as kernel, using the implementation in SciKit learn machine learning library [47] (values for $\mathrm{C}=1$ and $\gamma=$ auto and the kernel functions were empirically selected using a grid search of hyper parameter values belonging to $C=\in\{1, \ldots, 10\}, \gamma=\in\{$ auto, scaled $\}$ and kernel $=\in\{\mathrm{RBF}$, linear, polynomial $\}$ on a sub-set of the training data). The data was first centered and normalized to create normally distributed features with a variance of the same order. Training and test were then performed using a subject independent K-fold cross-validation, i.e., for every test subject, the SVM was trained on all the other subjects. The combined SVM classification was performed in two steps, with the linear kernel SVM first classifying the video clips, resulting in a high precision for the High and Low engagement classes, but with a bias towards the Neutral class. In the second step, the clips classified as Neutral were therefore reclassified using the RBF kernel SVM, resulting in the combined classification results presented in Figure 9. The overall balanced accuracy, i.e., the average of the recall on each class [47], of 65\% for the combined model may be compared to the baseline (selecting the most common class) of $38 \%$ and the 
Table 7. Summary of How Different Information Sources Confirm Hypotheses (A), Identify Learners Who Have Low Activity or Emotional Engagement and Also Lower Post-session Ratings in Different Categories (B), and Sort the Robot Interaction Strategies with Respect to Engagement with Different Measures of Engagement $(\mathrm{C})$

\begin{tabular}{|c|c|c|c|c|c|c|c|c|c|}
\hline $\begin{array}{l}\text { Information } \\
\text { source }\end{array}$ & $\begin{array}{l}\text { Confirmed } \\
\text { hypotheses }\end{array}$ & 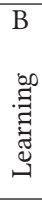 & 总 & 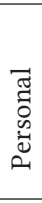 & 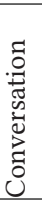 & 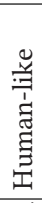 & $\begin{array}{l}\text { Number of } \\
\text { utterances }\end{array}$ & $\begin{array}{l}\text { Ratio of engaged } \\
\text { turns or duration }\end{array}$ & $\begin{array}{l}\text { Mean total length } \\
\text { (words) or duration } \\
\text { (seconds) }\end{array}$ \\
\hline $\begin{array}{l}\text { Verbal } \\
\text { Vocal (manual) } \\
\text { Vocal (auto) } \\
\text { Face (manual) } \\
\text { Face (auto) }\end{array}$ & $\begin{array}{l}\text { H, H1 } \\
\text { H, H2 } \\
\text { H, H2, H5 } \\
\text { H, H3 } \\
\text { H, H4-H5 }\end{array}$ & $\begin{array}{l}\sqrt{ } \\
\sqrt{ } \\
\sqrt{ }\end{array}$ & $\begin{array}{l}\sqrt{ } \\
\sqrt{ }\end{array}$ & $\begin{array}{l}\sqrt{ } \\
\sqrt{ }\end{array}$ & $\begin{array}{l}\sqrt{ } \\
\sqrt{ }\end{array}$ & $\begin{array}{l}\sqrt{ } \\
\sqrt{ } \\
\sqrt{ } \\
\sqrt{ } \\
\sqrt{ }\end{array}$ & $\begin{array}{l}\mathrm{Fa}^{*}<\mathrm{In}<\mathrm{Il}<\mathrm{Na} \\
\mathrm{Na}^{*}<\mathrm{In}<\mathrm{Il}<\mathrm{Fa}\end{array}$ & $\begin{array}{l}\mathrm{Na}^{*}<\mathrm{In}<\mathrm{Il}<\mathrm{Fa}^{*} \\
\mathrm{Na}^{*}<\mathrm{In}<\mathrm{Il}<\mathrm{Fa} \\
\mathrm{Fa}<\mathrm{Il}<\mathrm{Na}<\mathrm{In} \\
\mathrm{In}<\mathrm{Fa}\end{array}$ & $\begin{array}{l}\mathrm{Na}^{*}<\mathrm{In}<\mathrm{Il}<{ }^{*} \mathrm{Fa} \\
\mathrm{Na}^{*}<\mathrm{In}<\mathrm{Il}<\mathrm{Fa} \\
\mathrm{Fa}<\mathrm{Il}<\mathrm{In}<\mathrm{Na}\end{array}$ \\
\hline
\end{tabular}

In = Interviewer, $\mathrm{Il}=$ Interlocutor, $\mathrm{Fa}=$ Facilitator, $\mathrm{Na}=$ Narrator. ${ }^{*}<$ denotes significant differences between strategies.

inter-annotator agreement $\kappa=73 \%$, which could be interpreted as a measure of how good human observers were at determining the listener engagement level. The classification results are thus overall well above the baseline and rather close to the inter-rater agreement level, even if the recall for the Neutral class is low. When Figure 9 is considered it is moreover clear that the model is rather accurate (79\%) at identifying low-engaged listeners, and from an application point of view this is the most important, in order to be able to take adequate remedy actions, e.g., switching addressee to the disengaged participant. However, since the rate of false disengagement positives is high for, in particular, neutral listeners, the robot interaction strategy needs to include thresholds for the duration or number of turns addressing one learner before switching attention, to avoid the too frequent attention shifts that would result if the robot switches addressee as soon as it detects a disengaged listener.

\section{DISCUSSION}

Verbal, vocal, and facial information provide important information of how active and emotionally engaged a learner in robot-led conversation practice is, quantitatively through observations of the conversations and qualitatively linked to the learner's own ratings of the practice and the robot, which we consider as an indicator of subjective lower task or social engagement. Table 7 summarises how different information sources identified learners with low activity or emotional engagement who were also more negative towards the practice or the robot in the post-session ratings. These learners were, in particular, more negative regarding the robot's Friendliness and Human-likeness, which is similar to previous findings [41] that a robot that is not aware of the subjects' engagement is rated lower regarding how human-like and intelligent it is. We find that verbal activity, voice features, and facial expressions may indeed identify learners with indicators of lower social-task engagement (research question R). Since the personal relationship with the robot is important to maintain learner interest over time [35], such signals of low rapport with the robot need to be monitored. The results from the experiments on facial expressions are in line with previous studies that have determined that smiles and gaze can be used to determine engagement levels [12] and the results on correspondence between manual annotations and automatic labeling of voice features are similar to previous research results on, e.g., classification of anger, positive, and neutral in human-robot interaction $(37 \%-63 \%)$ [55].

Table 7 further summarizes research questions R1, R2, and R3 by indicating how the different robot interaction strategies are sorted with respect to level of activity and emotional engagement 
according to the different modalities and measures. Hypotheses H1, H2, and H3 were confirmed, with the exception of automatic classification of vocal arousal (H2), for which no statistically significant differences were found. It is natural that different measures may result in different rankings: the Narrator strategy resulted in many, but short, learner utterances, while the Facilitator on the contrary resulted in fewer but longer learner utterances. The rankings from verbal activity and manual annotations of vocal engagement are similar, except for the one based on a number of utterances. The difference is possible to explain by the fact that the former measures the total number and the latter only the engaged utterances, i.e., many of the Narrator utterances were not engaged. This is also the explanation for the difference in ranking between number of utterances and ratio for the automatic annotations of vocal engagement. The rankings from manual and automatic annotations of vocal engagement appear to differ substantially, but it should be noted firstly that many of the within-ranking differences are non-significant and secondly that the focus of the annotations differed, as explained in Section 6.

One question that could be discussed is if it is possible to identify a robot interaction strategy that in general is better for learner engagement. Over all conversations, it appears that Interlocutor or Facilitator were most successful at engaging learners. However, the differences between robot interaction strategies are over-shadowed by differences between learners and within learners. The learners did not only differ in how engaged they were in general, but also in their relative engagement with different robot interaction strategies. This illustrates the importance of identifying low-engaged learners rather than attempting to find a robot interaction strategy that would engage all learners. This also applies to the currently non-active listener in the learner pair (R4).

Finally, it was shown that a method for speech emotion detection (Section 6.3) and a standard machine learning implementation for classification based on facial features in video (Section 7.2) made reasonably similar judgement of learner engagement as manual annotations, despite the added complexity of interaction in an L2 and a multi-party setting in recordings in the wild (R5). The results may be improved upon by using more advanced machine learning algorithms, more training data and audiovisual recordings of higher quality. For speaker engagement several modalities may be combined to increase detection rate and robustness, but the different modalities textual content, amount and length of learner utterances, vocal features and facial expressions also complement each other (e.g., listener engagement can only be detected through video-based analysis) to find low learner engagement.

Two considerations must now be discussed, firstly how representative these results can be considered to be (Section 8.1) and secondly how the results may be used in future robot-led conversation practice (Section 8.2).

\subsection{Limitations}

The present study on learner engagement is, on one hand, performed on short interactions (1015 minutes) with first-time users, which signifies that the learners can be assumed to be more interested and engaged in the conversation than in long-term interactions with the robot [35]. On the other hand, most learners engaged in four conversations with the robot, and there was sometimes an overlap between topics addressed in conversations with the same user. Such repetitions of topics can be assumed to affect the learner's engagement negatively, which will penalise the rating of the later conversation, unrelated to robot interaction strategy. We mitigated this by rotating the order of the robot interaction strategies to the largest extent possible given the practical circumstances (see next paragraph). We did further not observe any bias in learner engagement depending on the conversation order (verbal: $p=0.13$ for NU, $p=0.79$ for TLU, $p=0.56$ for MLU, $\mathrm{p}=0.28$ for $\Phi_{\text {short }}$; vocal: $\mathrm{p}=0.54$ for $\Sigma, \mathrm{p}=0.19$ for $\Phi_{t}$; facial: $\mathrm{p}=0.97$ for $\phi_{H}, \mathrm{p}=0.33$ for $\phi_{L}, \mathrm{p}=$ 0.70 for $\delta$ for learner engagement, $\mathrm{p}=0.38$ for listener mean engagement level). 
As described in Section 4.2, the set-up for the user study was initially that every subject should experience all four robot interaction strategies and that the order of strategies should be rotated between subjects to ensure a balanced distribution between conversation numbers. Due to the subject drop-out and recruitment of new subjects, the dataset became unbalanced and betweenstrategy comparisons could hence risk being biased, but the effect should be marginal, as discussed in [24]. Moreover, the main research question is independent of robot interaction strategy and is hence unaffected by such potential bias.

The large variety in the learner group with respect to age, geographic origin, and linguistic and educational level is indeed representative of the target learner group and it is therefore intentional that the experiments were carried out with such a diverse group of learners, since the goal is that the robot should be able to adapt to the diversity in the learner group. However, since the withinsubject study design could not be followed entirely, differences might have been introduced due to subject variability rather than robot interaction strategy. To the extent possible we present the results in the figures as within-subject comparisons to allow for observations of differences between robot interaction strategies for the same subjects.

Manual annotation of engagement is subjective and categorical labels may not fully capture the variations. In this study, annotations were made either by one single labeler, thus ensuring similar labeling judgements for all utterances, but relying on one label per utterance, or with several labelers, with substantial agreement but also examples of inter-annotator variability when annotating some learners. Since human-human interaction also relies on subjective perception of the interlocutor, this may nevertheless be accepted also as a basis for human-robot interaction.

The two automatic classification studies were performed with standard methods and available software, without algorithmic improvements specific for the dataset or more advanced training of the models to suit the data. This means that the presented results may not show the full potential of machine learning detection of learner engagement. The listener engagement classification reached $65 \%$ balanced accuracy. At first glance, this is lower than the above $80 \%$ correct classification for 5-6 emotions that is often achieved [43], but is within the range of the state-of-the-art $(45 \%-$ $95 \%$ [43]), since the classification was of real engagement in the wild, with person-independent classification and less distinct facial emotional expressions that are more difficult to classify (as illustrated by the 0.73 inter-rater $\kappa$ agreement).

The video analysis experiments on learner and listener engagement were performed with, respectively, two and one of the robot interaction strategies, and it would be of interest to carry out an extended study with videos from all four strategies, in order to investigate if listener engagement is also influenced by the robot interaction strategy. Given the large amount of labeling and analysis required to determine when a learner is a listener and assess the level of engagement, this was saved for a future separate study. This study nevertheless showed that listener engagement may be estimated with sufficient accuracy from video images in the multi-party setting.

The robot design may influence learner engagement. On the one hand, the Furhat robotic head has unusually expressive-computer-animated-facial movements, even if it should be acknowledged that the full potential of the computer animation was not reached, since no additional emotional face expressions were overlayed the default visual speech synthesis and no attempts to match gaze were made (other than turning the head towards the learner). On the other hand, the Furhat robot is limited to the head and neck. Other body gestures, which are important in human spoken interaction, are hence excluded. We believe that the expressiveness of the face to some extent compensates for the lack of body gestures, but full-body robots may generate other levels or patterns of learner engagement even if the verbal interaction strategies would be the same.

It should finally be noted that the present analysis is made offline with previously collected and manually annotated data, whereas the aim is to be able to use these engagement signals 
autonomously during real-time interaction in order to adapt the conversations to better suit the learners. This would require some modifications. The verbal activity measures are currently based on number of transcribed words, but for real-time analysis it would be more feasible to instead use accumulated duration of learner utterances, to avoid that speech recognition errors influence the detection. The vocal and facial feature measures in this study use either manual annotations or autonomous detection and it is naturally only the latter that could be employed in a real practice setting. However, the experiments in Sections 6.3 and 7.2 indicate that the respective correspondence between the manual annotations and the autonomous methods ensure that the latter would be adequate for detection of low engagement. Concerning the complication of real-time performance, it is only natural that decisions regarding adaptation are based on a time window indicating low engagement over a couple of conversation turns rather than an immediate switch as soon as low engagement may have been detected, or else shifts in interaction strategy, topic or addressee risk being too frequent. This signifies that response times of the detection modules are less critical and it has moreover previously been demonstrated that robots may make other types of adaptations in real-time based on users' multimodal signals (e.g., [51]).

\subsection{Future Work}

This study has shown that objective measures of learner activity and engagement during the practice may be used to identify learners that have a more negative view of the practice and the robot and thus that and adaptation is required. The next step consists of determining what adaptation is required and implementing this adaptation.

For conversations with few or short learner utterances (Section 5.1), a suitable adaptation could be to either switch completely to a robot strategy that results in more learner activity (primarily Interviewer and Facilitator) or blend in parts of these strategies. For low engagement (Section 6.1) or low emotional arousal (Section 6.2), it would be appropriate to switch towards robot interaction strategies that have been found to increase the duration of engagement utterances (Interlocutor and Facilitator) or number of utterances with emotional arousal (Interviewer and Narrator). When it is detected that the non-active participant is becoming disengaged (Section 7.2), it is natural that the robot should try to involve that participant in the interaction, by switching addressee or robot interaction strategy to one involving both learners simultaneously.

Future work will focus on how to implement transitions between, and blending of, robot interaction strategy and investigations of how such adaptations influence learner engagement. In addition, future work will also target adaptation based on other communicative affective signals by the learners, such as learner uncertainty and confusion, which has been investigated in a follow-up study [15].

The overarching contribution of the present line of work is that detection of learner engagement enables the robot to alter its interaction to promote learner activity or balance it within learner pairs. In a recent study [27], we demonstrate that adaptive robot gaze behaviour can mediate participation imbalance (measured as the duration ratio of the speakers' utterances) within pairs with different linguistic levels: When the robot's gaze is adapted to focus more on the participant who contributes the least in the interaction, this participant becomes more active than with non-adaptive robot gaze. Adaptive robot behaviour may thus encourage lower-level learners to participate more in the practice, which is a key to their linguistic development. This effect will be strengthened when the robot is able to adapt to not only quantitative duration of learner participation, but also to qualitative emotional engagement. The identified measures and determined thresholds for low engagement with the included verbal, vocal and facial features can then be used to trigger changes in the robot's non-verbal (e.g., gaze as discussed above) or verbal (e.g., change of topic or conversation strategy) behaviour. 


\section{CONCLUSIONS}

The contributions of this study are that it showed that learners with low verbal activity and emotional engagement rated the practice and robot consistently lower; that the robot's interaction strategy influences the learners' activity and engagement; that listener engagement in the threeparty setting varied substantially both within conversations and between learners; that standard methods for detection of emotions in vocal or facial features can be applied to this multi-party real-world setting with reasonable correspondence with manual annotation, and finally, that verbal activity, acoustic voice features and facial expressions are important sources of information to identify learners with lower engagement for the practice.

Compliance with Ethical Standards: All subjects were briefed before the experiment using an informed consent form describing the experiment and the use and restricted distribution of the collected audio and video data. To comply with these privacy constraints, the datasets are not publicly available. However, subjects were asked to indicate in the informed consent form whether their data could be used for scientific presentations and all individuals displayed in figures in this article had agreed to this.

\section{ACKNOWLEDGMENTS}

The experiments in Section 5 use the transcriptions made by Gustav Melander and Robin Wänlund and Section 7 the engagement labels partly made by Eric Hartmanis and Patrik Ekman, as part of their respective BSc theses on linguistic analysis and video analysis of the robot-led conversations. The authors would like to thank the Swedish for Immigrant students at Hermods, Alvik, Stockholm for participating in the study, and finally the associate editor and the four reviewers for their many constructive suggestions on the manuscript.

\section{REFERENCES}

[1] Muneeb Ahmad, Omar Mubin, and Joanne Orlando. 2017. Adaptive social robot for sustaining social engagement during long-term children-robot interaction. International fournal of Human-Computer Interaction 33, 12 (03 2017), 1-20. DOI : https://doi.org/10.1080/10447318.2017.1300750

[2] Muneeb Ahmad, Omar Mubin, Suleman Shahid, and Joanne Orlando. 2019. Robot's adaptive emotional feedback sustains children's social engagement and promotes their vocabulary learning: A long-term child-robot interaction study. Adaptive Behavior 27, 4 (05 2019), 243-266. DOI : https://doi.org/10.1177/1059712319844182

[3] Samer Al Moubayed, Jonas Beskow, Gabriel Skantze, and Bjorn Granström. 2012. Furhat: A Back-projected Human-like Robot Head for Multiparty Human-Machine Interaction. Springer, 114-130.

[4] Samer Al Moubayed and Jill Lehman. 2015. Toward better understanding of engagement in multiparty spoken interaction with children. In Proceedings of the 2015 ACM on International Conference on Multimodal Interaction. Association for Computing Machinery, New York, NY, 211-218. DOI : https://doi.org/10.1145/2818346.2820733

[5] Ivon Arroyo, David Cooper, Winslow Burleson, Beverly Woolf, Kasia Muldner, and Robert Christopherson. 2009. Emotion sensors go to school. Frontiers in Artificial Intelligence and Applications 200, 1 (1 2009), 17-24. DOI : https: //doi.org/10.3233/978-1-60750-028-5-17

[6] Tadas Baltrusaitis, Peter Robinson, and Louis-Philippe Morency. 2016. OpenFace: An open source facial behavior analysis toolkit. In Proceedings of the 2016 IEEE Winter Conference on Applications of Computer Vision . 1-10.

[7] Atef Ben Youssef, Giovanna Varni, Slim Essid, and Chloé Clavel. 2019. On-the-fly detection of user engagement decrease in spontaneous human-robot interaction using recurrent and deep neural networks. International fournal of Social Robotics 11 (9 2019), 815-828. DOI : https://doi.org/10.1007/s12369-019-00591-2

[8] Timothy Bickmore, Daniel Schulman, and Langxuan Yin. 2010. Maintaining engagement in long-term interventions with relational agents. Applied Artificial Intelligence 24, 6 (7 2010), 648-666. DOI : https://doi.org/10.1080/08839514.2010. 492259

[9] Nigel Bosch, Sidney D’Mello, Ryan Baker, Jaclyn Ocumpaugh, Valerie Shute, Matthew Ventura, Lubin Wang, and Weinan Zhao. 2015. Automatic detection of learning-centered affective states in the wild. In Proceedings of the 20th International Conference on Intelligent User Interfaces . Association for Computing Machinery, New York, NY, 379-388. DOI : https://doi.org/10.1145/2678025.2701397 
[10] Felix Burkhardt, Astrid Paeschke, M. Rolfes, Walter Sendlmeier, and Benjamin Weiss. 2005. A database of German emotional speech. In Proceedings of the 9th European Conference on Speech Communication and Technology, Vol. 5. 1517-1520.

[11] Nick Campbell. 2004. Accounting for voice-quality variation. In Proceedings of the Speech Prosody. 217-220.

[12] Ginevra Castellano, Iolanda Leite, André Pereira, Carlos Martinho, Ana Paiva, and Peter Mcowan. 2009. Affect recognition for interactive companions: Challenges and design in real world scenarios. fournal on Multimodal User Interfaces 3 (3 2009), 89-98. DOI : https://doi.org/10.1007/s12193-009-0033-5

[13] L. J. Corrigan, Christopher Peters, Ginevra Castellano, Fotis Papadopoulos, Aidan Jones, Shweta Bhargava, Srini Janarthanam, Helen Hastie, Amol Deshmukh, and Ruth Aylett. 2013. Social-task engagement: Striking a balance between the robot and the task. In Proceedings of the Embodied Communication of Goals and Intentions Workshop. 1-7.

[14] Albert Costa, Martin J. Pickering, and Antonella Sorace. 2008. Alignment in second language dialogue. Language and Cognitive Processes 23, 4 (2008), 528-556. DOI : https://doi.org/10.1080/01690960801920545

[15] Ronald Cumbal, José Lopes, and Olov Engwall. 2020. Uncertainty in robot assisted second language conversation practice. In Proceedings of the Companion of the 2020 ACM/IEEE International Conference on Human-Robot Interaction. ACM/IEEE, Cambridge, United Kingdom, 171-173. DOI : https://doi.org/10.1145/3371382.3378306

[16] Mirjam de Haas, Paul Vogt, and Emiel Krahmer. 2020. The effects of feedback on children's engagement and learning outcomes in robot-assisted second language learning. Frontiers in Robotics and AI 7 (8 2020), 101. DOI : https://doi.org/ 10.3389/frobt.2020.00101

[17] Marc R. de Lepper, Maria Woolverton, Donna L. Mumme, and Jean-Luc Gurtner. 1993. Motivational Techniques of Expert Human Tutors: Lessons for the Design of Computer-based Tutors. Lawrence Erlbaum Associates, Inc., 75-105.

[18] Laurence Devillers and Laurence Vidrascu. 2006. Real-life emotions detection with lexical and paralinguistic cues on human-human call center dialogs. In Proceedings of the 9th International Conference on Spoken Language Processing. Pittsburgh, 801-804.

[19] L. Devillers and L. Vidrascu. 2007. Real-Life Emotion Recognition in Speech. Springer, Berlin, 34-42.

[20] Sidney D’Mello. 2013. A selective meta-analysis on the relative incidence of discrete affective states during learning with technology. Journal of Educational Psychology 105, 4 (11 2013), 1082-1099. DOI : https://doi.org/10.1037/a0032674

[21] Paul Ekman. 1999. Basic Emotions. John Wiley \& Sons, Chapter 3, 45-68.

[22] Stefan Engeser and Falko Rheinberg. 2008. Flow, performance and moderators of challenge-skill balance. Motivation and Emotion 32 (9 2008), 158-172. DOI : https://doi.org/10.1007/s11031-008-9102-4

[23] Olov Engwall and José Lopes. 2020. Interaction and collaboration in robot-assisted language learning for adults. Computer Assisted Language Learning (2020), 1-37. DOI : https://doi.org/10.1080/09588221.2020.1799821

[24] Olov Engwall, José Lopes, and Anna Åhlund. 2020. Robot interaction styles for conversation practice in second language learning. International fournal of Social Robotics 13 (2020), 1-26. DOI: https://doi.org/10.1007/s12369-02000635-y

[25] Olov Engwall, José Lopes, Roland Cumbal, Gustav Berndtson, Ruben Lindström, Patrik Ekman, Eric Hartmanis, Emelie Jin, Ella Johnston, Michael Mekonnen, and Gara Tahir. Accepted. Learner and Teacher Perspectives on Robot-led L2 Conversation Practice. ReCALL (Accepted).

[26] Mary Ellen Foster, Andre Gaschler, and Manuel Giuliani. 2017. Automatically classifying user engagement for dynamic multi-party human-robot interaction. International fournal of Social Robotics 9 (7 2017). DOI : https://doi.org/10.1007/ s12369-017-0414-y

[27] Sarah Gillet, Ronald Cumbal, André Pereira, José Lopes, Olov Engwall, and Iolanda Leite. 2021. Robot gaze can mediate participation imbalance in groups with different skill levels. In Proceedings of the 2021 ACM/IEEE International Conference on Human-Robot Interaction. Association for Computing Machinery, New York, NY, 303-311. DOI : https://doi.org/10.1145/3434073.3444670

[28] G. Gordon, S. Spaulding, J. K. Westlund, J. J. Lee, L. Plummer, M. Martinez, M. Das, and C. Breazeal. 2016. Affective personalization of a social robot tutor for children's second language skills. In Proceedings of the 13th AAAI Conference on Artificial Intelligence. Palo Alto, 3951-3957.

[29] Joakim Gustafson and Daniel Neiberg. 2010. Prosodic cues to engagement in non-lexical response tokens in Swedish. In Proceedings of DiSS-LPSS Joint Workshop.

[30] Kevin Hallgren. 2012. Computing inter-rater reliability for observational data: An overview and tutorial. Tutorials in Quantitative Methods for Psychology 8, 1 (7 2012), 23-34. DOI : https://doi.org/10.20982/tqmp.08.1.p023

[31] Ehsan Hoque, Rosalind Picard, Daniel McDuff, and Mohammed Hoque. 2012. Exploring temporal patterns in classifying frustrated and delighted smiles. In IEEE Transactions on Affective Computing 3, 3 323-334.

[32] Takamasa Iio, Ryota Maeda, Kohei Ogawa, Yuichiro Yoshikawa, Hiroshi Ishiguro, Kaori Suzuki, Tomohiro Aoki, Miharu Maesaki, and Mika Hama. 2018. Improvement of Japanese adults' English speaking skills via experiences speaking to a robot. Journal of Computer Assisted Learning 35, 2 (11 2018), 228-245. DOI : https://doi.org/10.1111/jcal 12325 
[33] A. Jain and D. Zongker. 1997. Feature selection: Evaluation, application, and small sample performance. IEEE Transactions on Pattern Analysis and Machine Intelligence 19, 2 (1997), 153-158.

[34] T. Kanda, T. Hirano, D. Eaton, and H. Ishiguro. 2004. Interactive robots as social partners and peer tutors for children: A field trial. Human-Computer Interaction 19, 1 (2004), 61-84.

[35] T. Kanda, R. Sato, N. Saiwaki, and H. Ishiguro. 2007. A two-month field trial in an elementary school for long-term human-robot interaction. IEEE Transactions on Robotics 23, 5 (2007), 962-971.

[36] Ashish Kapoor, Winslow Burleson, and Rosalind Picard. 2007. Automatic prediction of frustration. International fournal of Human-Computer Studies 65, 8 (2007), 724-736. DOI : https://doi.org/10.1016/j.jhcs.2007.02.003

[37] A. Khalifa, T. Kato, and S. Yamamoto. 2017. Measuring effect of repetitive queries and implicit learning with joining-in type robot assisted language learning system. In Proceedings of the ISCA Workshop on Speech and Language Technology in Education. Stockholm, Sweden, 13-17.

[38] David Klotz, Johannes Wienke, Julia Peltason, Britta Wrede, Sebastian Wrede, Vasil Khalidov, and Jean-Marc Odobez. 2011. Engagement-based multi-party dialog with a humanoid robot. In Proceedings of the SIGDIAL 2011 Conference: 12th Annual Meeting of the Special Interest Group on Discourse and Dialogue. 341-343.

[39] J. Richard Landis and Gary G. Koch. 1977. The measurement of observer agreement for categorical data. Biometrics 33, 1 (1977), 159-174.

[40] Iolanda Leite, Marissa Mccoy, Daniel Ullman, Nicole Salomons, and Brian Scassellati. 2015. Comparing models of disengagement in individual and group interactions. In Proceedings of the ACM/IEEE International Conference on HumanRobot Interaction, Vol. 2015. 99-105 DOI : https://doi.org/10.1145/2696454.2696466

[41] Liyuan Li, Qianli Xu, Gang Wang, Xinguo Yu, Yeow Tan, and Haizhou Li. 2015. Visual perception based engagement awareness for multiparty human-robot interaction. International fournal of Humanoid Robotics 12, 4 (6 2015), 1550019 DOI : https://doi.org/10.1142/S021984361550019X

[42] Michael H. Long. 1983. Native speaker/non-native speaker conversation and the negotiation of comprehensible input1. Applied Linguistics 4, 2 (7 1983), 126-141. DOI : https://doi.org/10.1093/applin/4.2.126

[43] Dhwani Mehta, Mohammad Faridul Haque Siddiqui, and Ahmad Javaid. 2018. Facial emotion recognition: A survey and real-world user experiences in mixed reality. Sensors 18, 2 (2018), 416 pages. DOI : https://doi.org/10.3390/ s18020416

[44] Emily Mower Provost, David Feil-Seifer, Maja Mataric, and Shrikanth Narayanan. 2007. Investigating implicit cues for user state estimation in human-robot interaction using physiological measurements. In Proceedings of the 16th IEEE International Symposium on Robot and Human Interactive Communication. 1125-1130. DOI : https://doi.org/10.1109/ ROMAN.2007.4415249

[45] Catharine Oertel, Kenneth Funes Mora, Joakim Gustafson, and Jean-Marc Odobez. 2015. Deciphering the silent participant: On the use of audio-visual cues for the classification of listener categories in group discussions. In Proceedings of the 2015 ACM on International Conference on Multimodal Interaction. 107-114. DOI : https://doi.org/10.1145/2818346 2820759

[46] Catharine Oertel, Stefan Scherer, and Nick Campbell. 2011. On the use of multimodal cues for the prediction of degrees of involvement in spontaneous conversation. In Proceedings of the INTERSPEECH. 1541-1544.

[47] F. Pedregosa, G. Varoquaux, A. Gramfort, V. Michel, B. Thirion, O. Grisel, M. Blondel, P. Prettenhofer, R. Weiss, V. Dubourg, J. Vanderplas, A. Passos, D. Cournapeau, M. Brucher, M. Perrot, and E. Duchesnay. 2011. Scikit-learn: Machine learning in python. Journal of Machine Learning Research 12 (2011), 2825-2830.

[48] Christopher Peters, Stylianos Asteriadis, Kostas Karpouzis, and Etienne de Sevin. 2008. Towards a real-time gaze-based shared attention for a virtual agent. In Proceedings of the Workshop on Affective Interaction in Natural Environments, ACM International Conference on Multimodal Interfaces.

[49] I. Poggi. 2007. Mind, Hands, Face and Body: A Goal and Belief View of Multimodal Communication. Weidler.

[50] Charles Rich, Brett Ponsleur, Aaron Holroyd, and Candace Sidner. 2010. Recognizing engagement in human-robot interaction. In Proceedings of ACM/IEEE International Conference on Human-Robot Interaction. 375-382. DOI : https: //doi.org/10.1145/1734454.1734580

[51] Hannes Ritschel, Tobias Baur, and Elisabeth André. 2017. Adapting a Robot's linguistic style based on socially-aware reinforcement learning. In Proceedings of the 26th IEEE International Symposium on Robot and Human Interactive Communication. 378-384. DOI : https://doi.org/10.1109/ROMAN.2017.8172330

[52] Arman Savran, Houwei Cao, Miraj Shah, Ani Nenkova, and Ragini Verma. 2012. Combining video, audio and lexical indicators of affect in spontaneous conversation via particle filtering. In Proceedings of the ACM International Conference on Multimodal Interaction. 485-492. DOI : https://doi.org/10.1145/2388676.2388781

[53] Thorsten Schodde, Laura Hoffmann, Sonja Stange, and Stefan Kopp. 2019. Adapt, explain, engage - a study on how social robots can scaffold second-language learning of children. ACM Transactions on Human-Robot Interaction 9, 1 (2019), 1-27. DOI : https://doi.org/10.1145/3366422 
[54] Candace Sidner, Cory Kidd, Christopher Lee, and Neal Lesh. 2004. Where to look: A study of human-robot engagement. In Proceedings of the 9th International Conference on Intelligent User Interfaces. 78-84. DOI : https://doi.org/10.1145/ 964442.964458

[55] Marie Tahon, Agnes Delaborde, and Laurence Devillers. 2011. Real-life emotion detection from speech in human-robot interaction: Experiments across diverse corpora with child and adult voices. In Proceedings of the INTERSPEECH. 31213124 .

[56] Sharon Unsworth. 2008. Comparing child L2 development with adult L2 development: How to measure L2 proficiency. In Current Trends in Child Second Language Acquisition: A Generative Perspective, Belma Haznedar and Elena Gavruseva (Eds.), 301-333, John Benjamins Publishing Company.

[57] Amy Warriner, Victor Kuperman, and Marc Brysbaert. 2013. Norms of valence, arousal, and dominance for 13,915 English lemmas. Behavior Research Methods 45 (2 2013), 1191-1207. DOI : https://doi.org/10.3758/s13428-012-0314-x

[58] Britta Wrede and Elizabeth Shriberg. 2003. Relationship between dialogue acts and hot spots in meetings. In Proceedings of the 2003 IEEE Workshop on Automatic Speech Recognition and Understanding (IEEE Cat. No. 03EX721). 180-185. DOI : https://doi.org/10.1109/ASRU.2003.1318425

[59] Mohammed Yeasin, Baptiste Bullot, and Rajeev Sharma. 2006. Recognition of facial expressions and measurement of levels of interest from video. IEEE Transactions on Multimedia 8, 3 (7 2006), 500-508. DOI: https://doi.org/10.1109/ TMM.2006.870737

[60] Chen Yu, Paul M. Aoki, and Allison Woodruff. 2004. Detecting user engagement in everyday conversations. In Proceedings of 8th International Conference on Spoken Language Processing, Vol. 2. Jeju Island, Republic of Korea, 1329-1332.

[61] Zerrin Yumak, Jianfeng Ren, Nadia Thalmann, and Junsong Yuan. 2014. Tracking and fusion for multiparty interaction with a virtual character and a social robot. In Proceedings of the SIGGRAPH Asia 2014 Autonomous Virtual Humans and Social Robot for Telepresence. DOI : https://doi.org/10.1145/2668956.2668958

[62] Zhihong Zeng, Maja Pantic, Glenn Roisman, and Thomas Huang. 2009. A survey of affect recognition methods: Audio, visual, and spontaneous expressions. IEEE Transactions on Pattern Analysis and Machine Intelligence 31, 1 (2 2009), 3958. DOI : https://doi.org/10.1109/TPAMI.2008.52

Received August 2020; revised October 2021; accepted November 2021 